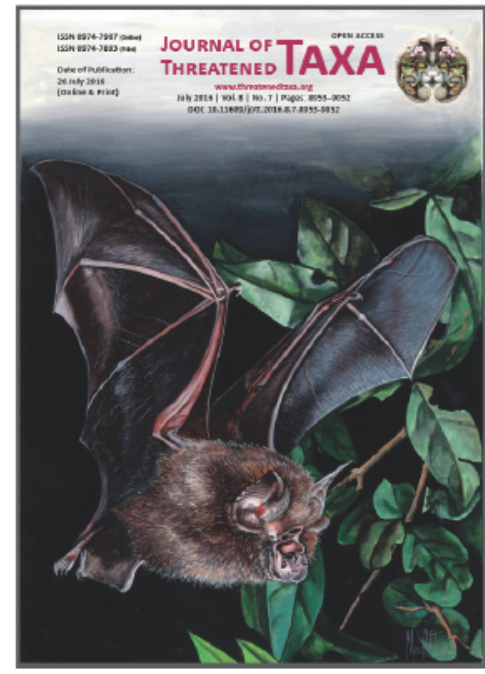

Journal of Threatened Taxa

The international journal of conservation and taxonomy

www.threatenedtaxa.org

ISSN 0974-7907 (Online) I ISSN 0974-7893 (Print)

\title{
REVIEW
}

\section{AN UPDATED CHECKLIST OF SHRIMPS ON THE INDIAN COAST}

Vijay Kumar Deepak Samuel, Chemmencheri Ramakrishnan Sreeraj, Pandian Krishnan, Chermapandi Parthiban, Veeramuthu Sekar, Kanagaraj Chamundeeswari, Titus Immanuel, Patro Shesdev, Ramachandran Purvaja \& Ramachandran Ramesh

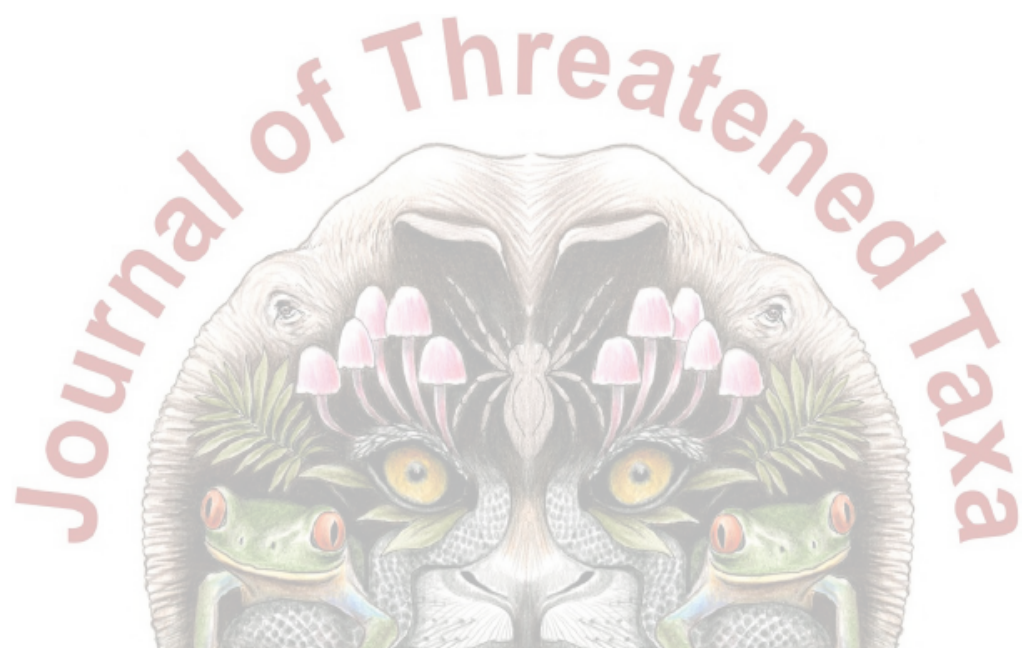

For Focus, Scope, Aims, Policies and Guidelines visit http://threatenedtaxa.org/About_JoTT.asp For Article Submission Guidelines visit http://threatenedtaxa.org/Submission_Guidelines.asp For Policies against Scientific Misconduct visit http://threatenedtaxa.org/JoTT_Policy_against_Scientific_Misconduct.asp For reprints contact <info@threatenedtaxa.org>

\section{Partner}

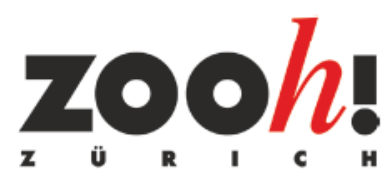

Publisher/Host

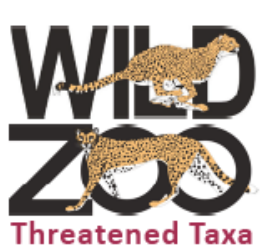





\section{AN UPDATED CHECKLIST OF SHRIMPS ON THE INDIAN COAST}

\author{
Vijay Kumar Deepak Samuel ${ }^{1}$, Chemmencheri Ramakrishnan Sreeraj ${ }^{2}$, Pandian Krishnan ${ }^{3}$, \\ Chermapandi Parthiban ${ }^{4}$, Veeramuthu Sekar ${ }^{5}$, Kanagaraj Chamundeeswari ${ }^{6}$, \\ Titus Immanuel ${ }^{7}$, Patro Shesdev ${ }^{8}$, Ramachandran Purvaja ${ }^{9}$ \& Ramachandran Ramesh $^{10}$
}

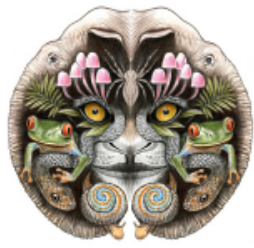

ISSN 0974-7907 (Online) ISSN 0974-7893 (Print)

OPEN ACCESS Anna University Campus, Chennai, Tamil Nadu 600025, India

${ }^{7}$ Central Island Agricultural Research Institute, Post Box No: 181, Port Blair 744101, Andaman \& Nicobar Island, India

${ }^{1}$ deepakocean@gmail.com, ${ }^{2}$ crsreeraj@gmail.com, ${ }^{3}$ krishnanars@yahoo.com (corresponding author),

${ }^{4}$ pcsparthiban@gmail.com, ${ }^{5}$ sekarveera15@gmail.com, ${ }^{6}$ cham mercy@yahoo.co.in,

7titusimmanuel@gmail.com, ${ }^{8}$ shesdevpatro@yahoo.com, ${ }^{9}$ purvaja.ramachandran@gmail.com,

${ }^{10}$ rramesh_au@yahoo.com

Abstract: This study reports an updated checklist of marine shrimps found along the Indian coast, including the Lakshadweep and the Andaman \& Nicobar Islands. A total of 364 species classified under 128 genera belonging to the order Decapoda is reported, thus adding 27 species to the existing checklist of 337 species. Marine shrimps are classified under two suborders of the order Decapoda, viz., Dendrobranchiata and Pleocyemata, and the two suborders account for $155(42.6 \%)$ and 209 species $(57.4 \%)$ of these 364 species, respectively. Pleocyemata is represented by three infraorders, viz., Axiidea, Caridea and Stenopodidea, while Caridea has a maximum of 199 reported species. Among the 12 superfamilies, Penaeoidea contributed to $38.13 \%$ (135 species) followed by Paleaemonidea with $18.07 \%$ (64 species). All other superfamilies were found to contribute less than $12 \%$. Superfamilies, Bresilloidea and Psalidopodoidea had only single species representatives $(0.28 \%$ each). The final list was compiled after reviewing all existing literature including monographs, catalogues, checklists, websites and fishery reports. The scientific names were validated with the World Register of Marine Species (WoRMS) database. A total of 25 issues were identified from the previous checklist out of which 19 species have been updated with the correct, accepted names and six species have been removed from the previous list.

Keywords: Checklist, Decapoda, Dendrobranchiata, India, Pleocyemata, shrimp.

DOI: http://dx.doi.org/10.11609/jott.2628.8.7.8977-8988 | ZooBank: urn:Isid:zoobank.org:pub:E549A41C-75F8-4506-9949-5BF46C7E7CB2

Editor: Stephen C. Weeks, The University of Akron, Ohio, USA.

Date of publication: 26 July 2016 (online \& print)

Manuscript details: Ms \# 2628 | Received 18 March 2016 | Final received 06 July 2016 | Finally accepted 18 July 2016

Citation: Samuel, V.K.D., C.R. Sreeraj, P. Krishnan, C. Parthiban, V. Sekar, K. Chamundeeswari, T. Immanuel, P. Shesdev, R. Purvaja \& R. Ramesh (2016). An updated checklist of shrimps on the Indian coast. Journal of Threatened Taxa 8(7): 8977-8988; http://dx.doi.org/10.11609/jott.2628.8.7.8977-8988

Copyright: @ Samuel et al. 2016. Creative Commons Attribution 4.0 International License. JoTT allows unrestricted use of this article in any medium, reproduction and distribution by providing adequate credit to the authors and the source of publication.

Funding: National Centre for Sustainable Coastal Management, Ministry of Environment, Forest and Climate Change, Chennai.

Conflict of Interest: The authors declare no competing interests.

For Tamil abstract, Author Contribution \& Author Details see end of this article.

Acknowledgements: The study was undertaken as a part of the research study on "Coastal and Marine Biodiversity Integration Network (CoMBINe)", by the Coastal and Marine Resources Conservation Division, National Centre for Sustainable Coastal Management, Chennai.

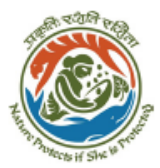

NCSCM

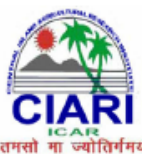




\section{INTRODUCTION}

Crustacean fishery as a whole accounts for more than $7 \%$ of the total produced through aquaculture. Crustaceans also play an important role in the economy by contributing more than $60 \%$ of the marine products exported (FAO 2014). Shrimp fishery is both an important food resource and a major economic resource for India. Shrimps account for $30 \%$ of the export of marine products in weight and $64 \%$ of the revenue generated from export of marine products (Anonymous 2014). Shrimps are the single most important marine food resource that is of the highest commercial value. There are several other shrimp species that may not be of commercial importance but form an integral part of the food chain of tropical marine ecosystems. Shrimps are a highly diverse group falling under the order Decapoda containing species that are widely distributed in marine, brackish, estuarine and freshwater realms. The majority of shrimp species occupy shallow or moderately deep sea water and are generally benthic, thriving on a variety of substrata such as rock, mud, peat, sand, fragments of shells or a mixture of these materials.

Studies of Decapoda date back to the end of the $19^{\text {th }}$ century, when exploratory surveys were carried out by the Royal Indian Marine Survey Ship (RIMS) 'Investigator' (Wood-Mason \& Alcock 1891; Alcock \& Anderson 1894, 1899; Alcock 1901; Kemp 1925). The first comprehensive catalogue of Indian deep sea Crustacea (Decapoda, Macrura and Anomala) was compiled by Alcock (1901). This was followed by several works from extensive surveys of shrimp resources in different regions of the Indian coast (e.g., Kunju 1960; George 1966; Muthu \& George 1971; Mohamed \& Suseelan 1973; Silas \& Muthu 1976; Thomas 1979; Rao 1984; Ravindranath 1989).

A total of 118 commercial shrimp species have been recorded by Suseelan (1996) that included 66 Penaeoid, 5 Sergestoid and 47 species of Caridean shrimps. Fortysix species of marine Palaemonid shrimps were recorded from the Indian seas by Jayachandran (2005) while, Karuppasamy et al. (2006) recorded 29 species of pelagic shrimps belonging to 19 genera and 11 families from the deep scattering layer (DSL) of the eastern Arabian Sea. Description of 84 Indian penaeid shrimps, along with their economic value and geographical distribution, were dealt with in detail by Kathirvel et al. (2007). Kurup et al. (2008) recorded 11 species of deep sea shrimps on the Kerala coast. A new gnathophyllid species was recorded from Lakshadweep waters by Prakash et al. (2011). Jayachandran (2010) listed 59 species of palaemonid shrimps with taxonomic status, state wise distribution and their conservation. Shanis et al. (2012) made a checklist of 24 pandalid species belonging to six genera from the Indian waters. Radhakrishnan et al. (2012) published an annotated checklist of the penaeoid, sergestoid, stenopodid and caridean shrimps of India. In their work, a total of 437 species were recorded out of which, 343 species were marine forms and 94 freshwater. This is the latest checklist available for the shrimps along the Indian coasts. Rajakumaran \& Vaseeharan (2014) surveyed shrimp diversity and exploitation of family Penaeidae from the southeastern coast of India and listed 59 species.

A knowledge of biodiversity will immensely help in the management of marine ecosystems and its resources. Regularly updated checklists on regional biodiversity would effectively aid in formulating proper and efficient conservation plans which require frequent/ regular surveys and timely publication updates. A recent and updated checklist of the shrimp fauna of the Indian waters is unavailable but is also most essential. The current work was carried out with an intention to produce a taxonomically verified checklist of the shrimps recorded along the Indian coast, which would be a base document for further studies.

\section{METHODS}

The checklist was prepared based on the collection of information from available published/reported literature, such as research articles, monographs, manuals, books, species checklists and technical reports. The scientific names were validated with the WoRMS (World Register of Marine Species) database for taxonomic status (accepted/status unknown, emendations, synonyms, alternate representations, nomen nudum, nomen dubium etc.) and the checklist was updated with currently accepted and valid names.

\section{RESULTS}

A total of 364 species under 128 genera of shrimp (Fig. 1 \& Table 1) are reported from the Indian coastal waters reviewing all previously published literature. Taxonomic hierarchy places species in this work under the order Decapoda that is divided into two suborders, namely Dendrobranchiata and Pleocyemata. Suborder Dendrobranchiata contains 155 species placed under two superfamilies, seven families and 41 


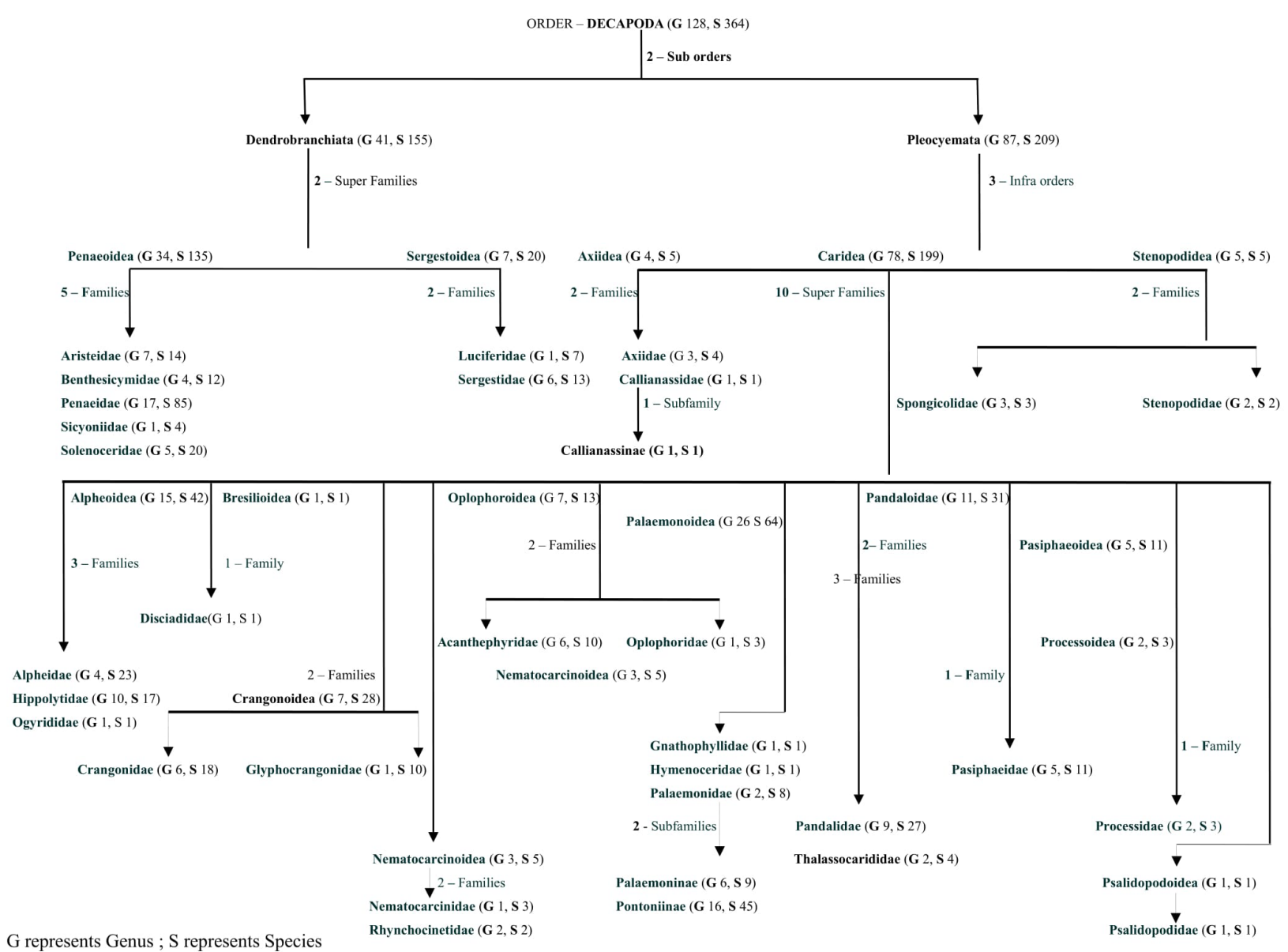

Figure 1. Flow chart representing the present classification and status of shrimps reported in Indian waters

genera contributing to $42.6 \%$ of the total 364 species. Suborder Pleocyemata contains 209 species included in three infraorders, 10 superfamilies, 22 families, three subfamilies and 87 genera with a contribution of $57.4 \%$ of the total 364 species. Family Penaeidae represents the greatest number of species, accounting for 85 species within 17 genera. This was followed by families Palaemonidae with 62 species under 24 genera, Pandalidae with 27 species under nine genera and Alpheidae with 23 species under four genera.

\section{DISCUSSION}

The diversity range of decapod crustaceans varies by spatial differences in environmental and oceanographic conditions, particularly by depth, bottom type and characteristics of the water masses (Abello et al. 1988). In India, shrimp species have been recorded from both shallow and deep waters.

The present work records a total of 364 species belonging to 128 genera after validating and updating data from earlier reports (Table 1). The highest contribution to species diversity was from the superfamily Penaeoidae (38.13\%) containing five families and 135 species (Fig. 1). Within the family Penaeidae, four genera, viz., Fenneropenaeus, Litopenaeus, Marsupenaeus and Melicertus, have been synonymised with the currently accepted genus Penaeus (Fransen \& De Grave 2015). Two species under the genera Parapenaeopsis, viz., P. hungerfordii and $P$. venusta, are accepted as Alcockpenaeopsis hungerfordii (Fransen 2015a) and Batepenaeopsis venusta (Fransen 2015b). The genus Plesiopenaeus is accepted as Cerataspis (Fransen 2015c) under the family Aristeidae. An addition of five species under two genera has been updated from the work of Shanis et al. (2012) which reported a total of 24 species under seven genera. Further, Thor maldivensis is a synonym of Thinora maldivensis (De Grave 2015) under the family Hippolytidae (Fransen 2015d). The work carried out by Radhakrishnan et al. (2012) has listed Notostomus sp. under the family 
Table 1. Checklist of valid names of shrimps from the Indian coast with information on their geographical distribution

\begin{tabular}{|c|c|c|c|c|c|}
\hline & Species name & Distribution & & Species name & Distribution \\
\hline \multirow{4}{*}{\multicolumn{3}{|c|}{$\begin{array}{l}\text { Kingdom: Animalia } \\
\text { Phylum: Arthropoda } \\
\text { Sub Phylum: Crustacea } \\
\text { Class: Malacostraca } \\
\text { Sub Class: Eumalacastraca } \\
\text { Super Order: Eucarida } \\
\text { Order: Decapoda } \\
\text { Sub Order: Dendrobranchiata } \\
\text { Super Family: Penaeoidea Rafinesque, } 1815\end{array}$}} & \multicolumn{3}{|c|}{ Family: Penaeidae Rafinesque, 1815} \\
\hline & & & 27 & $\begin{array}{l}\text { Alcockpenaeopsis hungerfordii } \\
\text { (Alcock, 1905) }\end{array}$ & Bay of Bengal \\
\hline & & & 28 & $\begin{array}{l}\text { Atypopenaeus compressipes } \\
\text { (Henderson, 1893) }\end{array}$ & Bay of Bengal \\
\hline & & & 29 & $\begin{array}{l}\text { Atypopenaeus stenodactylus } \\
\text { (Stimpson, 1860) }\end{array}$ & Northwest, southeast \\
\hline \multicolumn{3}{|c|}{ Family: Aristeidae Wood-Mason in Wood-Mason \& Alcock, 1891} & 30 & $\begin{array}{l}\text { Batepenaeopsis venusta (de } \\
\text { Man, 1907) }\end{array}$ & Southeast coast \\
\hline 1 & $\begin{array}{l}\text { Aristaeomorpha woodmasoni } \\
\text { Calman, } 1925\end{array}$ & $\begin{array}{l}\text { Andaman \& Nicobar Islands, } \\
\text { Arabian sea, Bay of Bengal }\end{array}$ & 31 & $\begin{array}{l}\text { Funchalia danae Burkenroad, } \\
1940\end{array}$ & Arabian Sea \\
\hline 2 & $\begin{array}{l}\text { Aristaeomorpha foliacea (Risso, } \\
\text { 1827) }\end{array}$ & $\begin{array}{l}\text { Arabian sea, Bay of Bengal, } \\
\text { Andaman \& Nicobar Islands }\end{array}$ & 32 & Funchalia villosa (Bouvier, 1905) & $\begin{array}{l}\text { West coast \& Andaman \& } \\
\text { Nicobar Islands }\end{array}$ \\
\hline 3 & $\begin{array}{l}\text { Aristaeomorpha woodmasoni } \\
\text { Calman, } 1925\end{array}$ & $\begin{array}{l}\text { Southwest and southeast coast, } \\
\text { Andaman \& Nicobar Islands }\end{array}$ & 33 & $\begin{array}{l}\text { Funchalia woodwardi Johnson, } \\
1868\end{array}$ & Southeast coast \\
\hline \multirow[t]{2}{*}{4} & \multirow{2}{*}{$\begin{array}{l}\text { Aristaeopsis edwardsiana } \\
\text { (Johnson, 1868) }\end{array}$} & \multirow{2}{*}{$\begin{array}{l}\text { Southwest, southeast, } \\
\text { Lakshadweep Islands, Andaman } \\
\text { \& Nicobar Islands }\end{array}$} & 34 & $\begin{array}{l}\text { Ganjampenaeopsis uncta (Alcock, } \\
\text { 1905) }\end{array}$ & Bay of Bengal \\
\hline & & & & Kishinouyepenaeopsis amicus & \\
\hline \multirow{2}{*}{5} & \multirow{2}{*}{ Aristeus alcocki Ramadan, 1938} & \multirow{2}{*}{$\begin{array}{l}\text { Southwest, Southeast, } \\
\text { Lakshadweep, Kerala }\end{array}$} & 30 & (V.C. Nguyên, 1971) & soutneast coast \\
\hline & & & & Kishinouyepenaeopsis & \\
\hline \multirow{2}{*}{6} & \multirow{2}{*}{$\begin{array}{l}\text { Aristeus semidentatus Spence } \\
\text { Bate, } 1881\end{array}$} & \multirow{2}{*}{$\begin{array}{l}\text { Southwest \& Andhra Pradesh } \\
\text { coast }\end{array}$} & 36 & maxillipedo (Alcock, 1905) & Soutneast coast \\
\hline & & & 37 & Megokris granulosus (Haswell, & Chennai, Kakinada \\
\hline \multirow{2}{*}{7} & \multirow{2}{*}{$\begin{array}{l}\text { Aristeus virilis (Spence Bate, } \\
\text { 1881) }\end{array}$} & \multirow{2}{*}{ Andaman \& Nicobar Islands } & 21 & 1879) & Cnennal, KaKınada \\
\hline & & & 38 & Megokris pescadoreensis & Southwest, Southeast \\
\hline \multirow[b]{2}{*}{8} & \multirow{2}{*}{$\begin{array}{l}\text { Cerataspis coruscans (Wood- } \\
\text { Mason in Wood-Mason \& Alcock, } \\
\text { 1891) }\end{array}$} & \multirow[b]{2}{*}{ Andaman \& Nicobar Islands } & 38 & (Schmitt, 1931) & sountroves, soutriedst \\
\hline & & & 39 & Megokris sedili (Hall, 1961) & $\begin{array}{l}\text { Southwest, southeast and } \\
\text { northeast }\end{array}$ \\
\hline 9 & $\begin{array}{l}\text { Cerataspis monstruosus Gray, } \\
1828\end{array}$ & Bay of Bengal & 40 & $\begin{array}{l}\text { Metapenaeopsis andamanensis } \\
\text { (Wood-Mason in Wood-Mason \& }\end{array}$ & Southwest, southeast, Andaman \\
\hline \multirow{2}{*}{10} & \multirow{2}{*}{$\begin{array}{l}\text { Hemipenaeus carpenteri Wood- } \\
\text { Mason \& Alcock, } 1891\end{array}$} & \multirow{2}{*}{ Minicoy, Bay of Bengal } & & Alcock, 1891) & \\
\hline & & & 41 & Metapenaeopsis barbata (De & South and northeast \\
\hline 11 & Hepomadus tener Smith, 1884 & Bay of Bengal & $4 \pm$ & Haan, 1844) & 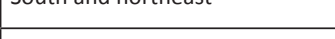 \\
\hline \multirow{2}{*}{12} & \multirow{2}{*}{$\begin{array}{l}\text { Pseudaristeus crassipes (Wood- } \\
\text { Mason in Wood-Mason \& Alcock, } \\
\text { 1891) }\end{array}$} & \multirow{2}{*}{$\begin{array}{l}\text { Lakshadweep Islands, Southwest } \\
\text { coast, Andaman \& Nicobar } \\
\text { Islands }\end{array}$} & 42 & $\begin{array}{l}\text { Metapenaeopsis ceylonica } \\
\text { Starobogatov, } 1972\end{array}$ & Kakinada \\
\hline & & & 43 & Metapenaeopsis commensalis & Lakshadweep Islands \\
\hline \multirow{2}{*}{13} & Pseudaristeus kathleenae Pérez & & & & \\
\hline & Farfante, 1987 & - & & Metapenaeopsis coniger (Wood- & Southwest, south and northeast, \\
\hline 14 & $\begin{array}{l}\text { Pseudaristeus protensus Pérez } \\
\text { Farfante, } 1987\end{array}$ & East and west coast & 44 & 1891) & Andaman \& Nicobar Islands \\
\hline Fan & : Benthesicymidae Wood-Mason in & Wood-Mason \& Alcock, 1891 & 45 & $\begin{array}{l}\text { Metapenaeopsis gaillardi } \\
\text { Crosnier, } 1991\end{array}$ & Southern India \\
\hline 15 & $\begin{array}{l}\text { Altelatipes carinatus (Smith, } \\
\text { 1884) }\end{array}$ & Lakshadweep & 46 & $\begin{array}{l}\text { Metapenaeopsis gallensis } \\
\text { (Pearson, 1905) }\end{array}$ & Chennai \\
\hline 16 & $\begin{array}{l}\begin{array}{l}\text { Bentheogennema intermedia } \\
\text { (Spence Bate, 1888) }\end{array} \\
\end{array}$ & - & 47 & $\begin{array}{l}\text { Metapenaeopsis hilarula (de } \\
\text { Man, 1911) }\end{array}$ & East and west coast \\
\hline 17 & $\begin{array}{l}\text { Bentheogennema pasithea (de } \\
\text { Man, 1907) }\end{array}$ & Arabian sea & 48 & $\begin{array}{l}\text { Metapenaeopsis mogiensis } \\
\text { Rathbun, } 1902\end{array}$ & $\begin{array}{l}\text { East and west coast, Andaman \& } \\
\text { Nicobar Islands }\end{array}$ \\
\hline 18 & $\begin{array}{l}\text { Benthesicymus armatus } \\
\text { MacGilchrist, } 1905\end{array}$ & - & 49 & $\begin{array}{l}\text { Metapenaeopsis novaeguineae } \\
\text { (Haswell, 1879) }\end{array}$ & Southeast coast \\
\hline 19 & $\begin{array}{l}\text { Benthesicymus bartletti Smith, } \\
1882\end{array}$ & - & 50 & $\begin{array}{l}\text { Metapenaeopsis palmensis } \\
\text { (Haswell, 1879) }\end{array}$ & Andaman \& Nicobar Islands \\
\hline 20 & $\begin{array}{l}\text { Benthesicymus investigatoris } \\
\text { Alcock \& Anderson, } 1899\end{array}$ & $\begin{array}{l}\text { Gulf of Mannar, Andaman \& } \\
\text { Nicobar Islands }\end{array}$ & 51 & $\begin{array}{l}\text { Metapenaeopsis philippi (Spence } \\
\text { Bate, 1881) }\end{array}$ & Southwest coast \\
\hline 21 & Gennadas bouvieri Kemp, 1909 & Bay of Bengal & 52 & $\begin{array}{l}\text { Metapenaeopsis stridulans } \\
\text { (Alcock, 1905) }\end{array}$ & $\begin{array}{l}\text { Northwest, south and northeast, } \\
\text { Andaman \& Nicobar Islands }\end{array}$ \\
\hline 22 & Gennadas parvus Spence Bate, & Arabian sea & & & \\
\hline 22 & $\begin{array}{l}1881 \\
\text { Gennadas propinquus Rathbun }\end{array}$ & Aldoldin Sed & 53 & $\begin{array}{l}\text { Metapenaeopsis toloensis Hall, } \\
1962\end{array}$ & Chennai \\
\hline 23 & $\begin{array}{l}\text { Gennadas propInquus Rathbun, } \\
1906\end{array}$ & Southwest and Southeast & 54 & $\begin{array}{l}\text { Metapenaeopsis wellsi Racek, } \\
1967\end{array}$ & Southeast coast \\
\hline 24 & Gennadas scutatus Bouvier, 1906 & Southwest coast & & & East and west coast, Andaman \& \\
\hline 25 & Gennadas sordidus Kemp, 1910 & Goa and Arabian sea & 55 & Edwards, 1837) & Nicobar Islands \\
\hline 26 & Gennadas tinayrei Bouvier, 1906 & - & 56 & $\begin{array}{l}\text { Metapenaeus alcocki M.J. George } \\
\& \text { Rao, } 1968\end{array}$ & Gulf of Kutch \\
\hline & & & 57 & $\begin{array}{l}\text { Metapenaeus anchistus (de Man, } \\
\text { 1920) }\end{array}$ & Southeast coast \\
\hline
\end{tabular}




\begin{tabular}{|c|c|c|}
\hline & Species name & Distribution \\
\hline 58 & $\begin{array}{l}\text { Metapenaeus brevicornis ( } \mathrm{H} . \\
\text { Milne Edwards, 1837) }\end{array}$ & $\begin{array}{l}\text { East and west coast, Andaman \& } \\
\text { Nicobar Islands }\end{array}$ \\
\hline 59 & $\begin{array}{l}\text { Metapenaeus dobsoni (Miers, } \\
\text { 1878) }\end{array}$ & $\begin{array}{l}\text { East and west coast, Andaman \& } \\
\text { Nicobar Islands }\end{array}$ \\
\hline 60 & $\begin{array}{l}\text { Metapenaeus elegans de Man, } \\
1907\end{array}$ & Andaman \& Nicobar Islands \\
\hline 61 & $\begin{array}{l}\text { Metapenaeus endeavouri } \\
\text { (Schmitt, 1926) }\end{array}$ & Kakinada, Andhra Pradesh \\
\hline 62 & $\begin{array}{l}\text { Metapenaeus ensis (De Haan, } \\
\text { 1844) }\end{array}$ & $\begin{array}{l}\text { South and northeast, Andaman \& } \\
\text { Nicobar Islands }\end{array}$ \\
\hline 63 & $\begin{array}{l}\text { Metapenaeus intermedius } \\
\text { (Kishinouye, 1900) }\end{array}$ & Andaman \& Nicobar Islands \\
\hline 64 & $\begin{array}{l}\text { Metapenaeus joyneri (Miers, } \\
1880 \text { ) }\end{array}$ & Southeast coast \\
\hline 65 & $\begin{array}{l}\text { Metapenaeus krishnatrii Silas \& } \\
\text { Muthu, } 1976\end{array}$ & Andaman \& Nicobar Islands \\
\hline 66 & $\begin{array}{l}\text { Metapenaeus kutchensis P.C. } \\
\text { George, M.J. George \& Rao, } 1963\end{array}$ & Northwest and northeast coast \\
\hline 67 & $\begin{array}{l}\text { Metapenaeus lysianassa (de } \\
\text { Man, 1888) }\end{array}$ & Southeast and northeast \\
\hline 68 & $\begin{array}{l}\text { Metapenaeus monoceros } \\
\text { (Fabricius, 1798) }\end{array}$ & East and west coast \\
\hline 69 & $\begin{array}{l}\text { Metapenaeus moyebi } \\
\text { (Kishinouye, 1896) }\end{array}$ & $\begin{array}{l}\text { Northeast, southwest \& } \\
\text { Andaman \& Nicobar Islands }\end{array}$ \\
\hline 70 & $\begin{array}{l}\text { Metapenaeus papuensis Racek \& } \\
\text { Dall, } 1965\end{array}$ & Southeast coast \\
\hline 71 & $\begin{array}{l}\text { Metapenaeus stebbingi Nobili, } \\
1904\end{array}$ & Gulf of Kutch \\
\hline 72 & $\begin{array}{l}\text { Mierspenaeopsis indica (Muthu, } \\
\text { 1972) }\end{array}$ & Southeast coast \\
\hline 73 & $\begin{array}{l}\text { Parapenaeopsis acclivirostris } \\
\text { Alcock, } 1905\end{array}$ & East and west coast \\
\hline 74 & $\begin{array}{l}\text { Parapenaeopsis cornuta } \\
\text { (Kishinouye, 1900) }\end{array}$ & $\begin{array}{l}\text { Northwest, southeast, northeast, } \\
\text { Andaman \& Nicobar Islands }\end{array}$ \\
\hline 75 & $\begin{array}{l}\text { Parapenaeopsis coromandelica } \\
\text { Alcock, } 1906\end{array}$ & Southeast coast \\
\hline 76 & $\begin{array}{l}\text { Parapenaeopsis gracillima Nobili, } \\
1903\end{array}$ & Southeast coast \\
\hline 77 & $\begin{array}{l}\text { Parapenaeopsis hardwickii } \\
\text { (Miers, 1878) }\end{array}$ & $\begin{array}{l}\text { Northwest, southeast and } \\
\text { northeast }\end{array}$ \\
\hline 78 & $\begin{array}{l}\text { Parapenaeopsis investigatoris } \\
\text { (Alcock \& Anderson, 1899) }\end{array}$ & Kerala \\
\hline 79 & $\begin{array}{l}\text { Parapenaeopsis longirostris } \\
\text { Chandra \& Bhattacharya, } 2004\end{array}$ & Odissa \\
\hline 80 & $\begin{array}{l}\text { Parapenaeopsis nana Alcock, } \\
1905\end{array}$ & South and northeast coast \\
\hline 81 & $\begin{array}{l}\text { Parapenaeopsis sculptilis (Heller, } \\
\text { 1862) }\end{array}$ & East and west coast \\
\hline 82 & $\begin{array}{l}\text { Parapenaeopsis stylifera }(\mathrm{H} . \\
\text { Milne Edwards, 1837) }\end{array}$ & $\begin{array}{l}\text { East and west coast, Andaman \& } \\
\text { Nicobar Islands }\end{array}$ \\
\hline 83 & $\begin{array}{l}\text { Parapenaeopsis tenella (Spence } \\
\text { Bate, 1888) }\end{array}$ & South and northeast \\
\hline 84 & $\begin{array}{l}\text { Parapenaeus fissuroides } \\
\text { fissuroides Crosnier, } 1986\end{array}$ & Southeast coast \\
\hline 85 & $\begin{array}{l}\text { Parapenaeus fissuroides indicus } \\
\text { Crosnier, } 1986\end{array}$ & Mangalore \\
\hline 86 & $\begin{array}{l}\text { Parapenaeus fissurus (Spence } \\
\text { Bate, 1881) }\end{array}$ & $\begin{array}{l}\text { Orissa, Andaman \& Nicobar } \\
\text { Islands }\end{array}$ \\
\hline 87 & $\begin{array}{l}\text { Parapenaeus investigatoris } \\
\text { Alcock \& Anderson, } 1899\end{array}$ & $\begin{array}{l}\text { Southwest, southeast, Andaman } \\
\text { \& Nicobar Islands }\end{array}$ \\
\hline 88 & $\begin{array}{l}\text { Parapenaeus lanceolatus Kubo, } \\
1949\end{array}$ & Southeast coast \\
\hline 89 & $\begin{array}{l}\text { Parapenaeus longipes Alcock, } \\
1905\end{array}$ & East and west coast \\
\hline 90 & $\begin{array}{l}\text { Parapenaeus sextuberculatus } \\
\text { Kubo, } 1949\end{array}$ & - \\
\hline
\end{tabular}

\begin{tabular}{|c|c|c|}
\hline & Species name & Distribution \\
\hline 91 & $\begin{array}{l}\text { Pelagopenaeus balboae (Faxon, } \\
\text { 1893) }\end{array}$ & Southwest coast \\
\hline 92 & $\begin{array}{l}\text { Penaeopsis jerryi Pérez Farfante, } \\
1979\end{array}$ & $\begin{array}{l}\text { Southwest, southeast and } \\
\text { Andaman \& Nicobar Islands }\end{array}$ \\
\hline 93 & $\begin{array}{l}\text { Penaeopsis rectacuta (Spence } \\
\text { Bate, 1881) }\end{array}$ & $\begin{array}{l}\text { Southwest, southeast and } \\
\text { Andaman \& Nicobar Islands }\end{array}$ \\
\hline 94 & $\begin{array}{l}\text { Penaeus canaliculatus (Olivier, } \\
\text { 1811) }\end{array}$ & $\begin{array}{l}\text { East and west coast, Minicoy, } \\
\text { Andaman \& Nicobar Islands }\end{array}$ \\
\hline 95 & $\begin{array}{l}\text { Penaeus hathor (Burkenroad, } \\
\text { 1959) }\end{array}$ & - \\
\hline 96 & $\begin{array}{l}\text { Penaeus indicus H. Milne } \\
\text { Edwards, } 1837\end{array}$ & $\begin{array}{l}\text { East and west coast, Andaman \& } \\
\text { Nicobar Islands }\end{array}$ \\
\hline 97 & $\begin{array}{l}\text { Penaeus japonicus Spence Bate, } \\
1888\end{array}$ & $\begin{array}{l}\text { East and west coast, Andaman \& } \\
\text { Nicobar Islands }\end{array}$ \\
\hline 98 & $\begin{array}{l}\text { Penaeus konkani (Chandra \& } \\
\text { Bhattacharya, 2003) }\end{array}$ & Goa, Malawan coast \\
\hline 99 & $\begin{array}{l}\text { Penaeus latisulcatus Kishinouye, } \\
1896\end{array}$ & $\begin{array}{l}\text { Northwest, southwest, } \\
\text { southeast, Lakshadweep Islands, } \\
\text { Andaman \& Nicobar Islands }\end{array}$ \\
\hline 100 & $\begin{array}{l}\text { Penaeus merguiensis de Man, } \\
1888\end{array}$ & $\begin{array}{l}\text { East and west coast, Andaman \& } \\
\text { Nicobar Islands }\end{array}$ \\
\hline 101 & $\begin{array}{l}\text { Penaeus monodon Fabricius, } \\
1798\end{array}$ & $\begin{array}{l}\text { East and west coast, Andaman \& } \\
\text { Nicobar Islands }\end{array}$ \\
\hline 102 & Penaeus penicillatus Alcock, 1905 & Northeast and northwest \\
\hline 103 & $\begin{array}{l}\text { Penaeus semisulcatus De Haan, } \\
1844\end{array}$ & $\begin{array}{l}\text { East and West coast, Andaman \& } \\
\text { Nicobar Islands }\end{array}$ \\
\hline 104 & $\begin{array}{l}\text { Penaeus silasi Muthu \& Motoh, } \\
1979\end{array}$ & Chennai \\
\hline 105 & $\begin{array}{l}\text { Penaeus similis (Chanda \& } \\
\text { Bhattacharya, 2002) }\end{array}$ & Arabian sea \\
\hline 106 & Penaeus vannamei Boone, 1931 & East \& West coast \\
\hline 107 & $\begin{array}{l}\text { Trachypenaeopsis minicoyensis } \\
\text { Thomas, } 1972\end{array}$ & Minicoy \\
\hline 108 & $\begin{array}{l}\text { Trachysalambria aspera (Alcock, } \\
1905 \text { ) }\end{array}$ & $\begin{array}{l}\text { Chennai, Orissa coast, Andaman } \\
\text { \& Nicobar Islands } \\
\end{array}$ \\
\hline 109 & $\begin{array}{l}\text { Trachysalambria curvirostris } \\
\text { (Stimpson, 1860) }\end{array}$ & East and west coast \\
\hline 110 & $\begin{array}{l}\text { Trachysalambria fulva (Dall, } \\
\text { 1957) }\end{array}$ & Chennai \\
\hline 111 & $\begin{array}{l}\text { Trachysalambria longipes } \\
\text { (Paul'son, 1875) }\end{array}$ & Southeast coast \\
\hline \multicolumn{3}{|c|}{ Family: Sicyoniidae Ortmann, 1898} \\
\hline 112 & Sicyonia fallax de Man, 1907 & - \\
\hline 113 & Sicyonia lancifer (Olivier, 1811) & Southwest and southeast \\
\hline 114 & $\begin{array}{l}\text { Sicyonia longicauda Rathbun, } \\
1906\end{array}$ & - \\
\hline 115 & $\begin{array}{l}\text { Sicyonia parajaponica Crosnier, } \\
2003\end{array}$ & Southwest and Southeast \\
\hline \multicolumn{3}{|c|}{ Family: Solenoceridae Wood-Mason in Wood-Mason \& Alcock, 1891} \\
\hline 116 & $\begin{array}{l}\text { Gordonella villosa (Alcock \& } \\
\text { Anderson, 1894) }\end{array}$ & Karwar and Minicoy \\
\hline 117 & $\begin{array}{l}\text { Hadropenaeus lucasii (Spence } \\
\text { Bate, 1881) }\end{array}$ & \\
\hline 118 & $\begin{array}{l}\text { Haliporus taprobanensis Alcock \& } \\
\text { Anderson, } 1899\end{array}$ & Gulf of Mannar and Kanyakumari \\
\hline 119 & Haliporus thetis Faxon, 1893 & - \\
\hline 120 & $\begin{array}{l}\text { Hymenopenaeus equalis (Spence } \\
\text { Bate, 1888) }\end{array}$ & \begin{tabular}{|l|} 
Southeast and southwest, \\
Andaman \& Nicobar Islands
\end{tabular} \\
\hline 121 & $\begin{array}{l}\text { Hymenopenaeus laevis (Spence } \\
\text { Bate, 1881) }\end{array}$ & Lakshadweep \\
\hline 122 & $\begin{array}{l}\text { Hymenopenaeus neptunus } \\
\text { (Spence Bate, 1881) }\end{array}$ & Bay of Bengal \\
\hline 123 & $\begin{array}{l}\text { Hymenopenaeus sewelli } \\
\text { Ramadan, } 1938\end{array}$ & - \\
\hline
\end{tabular}




\begin{tabular}{|c|c|c|}
\hline & Species name & Distribution \\
\hline 124 & $\begin{array}{l}\text { Solenocera alfonso Pérez } \\
\text { Farfante, } 1981\end{array}$ & Tuticorin \\
\hline 125 & $\begin{array}{l}\text { Solenocera alticarinata Kubo, } \\
1949\end{array}$ & - \\
\hline 126 & $\begin{array}{l}\text { Solenocera annectens (Wood- } \\
\text { Mason in Wood-Mason \& Alcock, } \\
\text { 1891) }\end{array}$ & Andaman \& Nicobarlslands \\
\hline 127 & Solenocera choprai Nataraj, 1945 & Southwest and east coast \\
\hline 128 & $\begin{array}{l}\text { Solenocera crassicornis (H. Milne } \\
\text { Edwards, 1837) }\end{array}$ & $\begin{array}{l}\text { East and west coast, Andaman \& } \\
\text { Nicobar Islands }\end{array}$ \\
\hline 129 & $\begin{array}{l}\text { Solenocera halli Starobogatov, } \\
1972\end{array}$ & $\begin{array}{l}\text { East and west coast, Andaman \& } \\
\text { Nicobar Islands }\end{array}$ \\
\hline 130 & $\begin{array}{l}\text { Solenocera hextii Wood-Mason \& } \\
\text { Alcock, } 1891\end{array}$ & East and west coast \\
\hline 131 & Solenocera koelbeli de Man, 1911 & Kerala, Andhra Pradesh \\
\hline 132 & $\begin{array}{l}\text { Solenocera melantho de Man, } \\
1907\end{array}$ & Southwest, northeast \\
\hline 133 & $\begin{array}{l}\text { Solenocera pectinata (Spence } \\
\text { Bate, 1888) }\end{array}$ & $\begin{array}{l}\text { Southwest coast \& Andhra } \\
\text { Pradesh }\end{array}$ \\
\hline 134 & $\begin{array}{l}\text { Solenocera pectinulata Kubo, } \\
1949\end{array}$ & West coast \\
\hline 135 & $\begin{array}{l}\text { Solenocera waltairensis M.J. } \\
\text { George \& Muthu, } 1970\end{array}$ & Kakinada, Visakhapatanam \\
\hline \multicolumn{3}{|c|}{$\begin{array}{l}\text { Super family: Sergestoidea Dana, } 1852 \\
\text { Family: Luciferidae De Haan, } 1849\end{array}$} \\
\hline 136 & Lucifer chacei Bowman, 1967 & $\begin{array}{l}\text { Arabian Sea, Lakshadweep } \\
\text { Islands, Bay of Bengal, Andaman } \\
\text { \& Nicobar Islands }\end{array}$ \\
\hline 137 & Lucifer faxoni Borradaile, 1915 & $\begin{array}{l}\text { Arabian Sea, Lakshadweep } \\
\text { Islands, Bay of Bengal, Andaman } \\
\& \text { Nicobar Islands }\end{array}$ \\
\hline 138 & Lucifer hanseni Nobili, 1905 & $\begin{array}{l}\text { Arabian Sea, Lakshadweep } \\
\text { Islands, Bay of Bengal, Andaman } \\
\text { \& Nicobar Islands }\end{array}$ \\
\hline 139 & Lucifer intermedius(Hansen, 1919 & $\begin{array}{l}\text { Arabian Sea, Lakshadweep } \\
\text { Islands, Bay of Bengal, Andaman } \\
\text { \& Nicobar Islands }\end{array}$ \\
\hline 140 & Lucifer orientalis Hansen, 1919 & $\begin{array}{l}\text { Arabian Sea, Lakshadweep } \\
\text { Islands, Bay of Bengal, Andaman } \\
\text { \& Nicobar Islands }\end{array}$ \\
\hline 141 & Lucifer penicillifer Hansen, 1919 & $\begin{array}{l}\text { Arabian Sea, Lakshadweep } \\
\text { Islands, Bay of Bengal, Andaman } \\
\& \text { Nicobar Islands }\end{array}$ \\
\hline 142 & $\begin{array}{l}\text { Lucifer typus H. Milne Edwards, } \\
1837\end{array}$ & $\begin{array}{l}\text { Arabian Sea, Lakshadweep } \\
\text { Islands,Bay of Bengal, Andaman } \\
\text { \& Nicobar Islands }\end{array}$ \\
\hline \multicolumn{3}{|c|}{ Family: Sergestidae Dana, 1852} \\
\hline 143 & Acetes erythraeus Nobili, 1905 & Southwest coast, east coast \\
\hline 144 & $\begin{array}{l}\text { Acetes indicus H. Milne Edwards, } \\
1830\end{array}$ & East and west coast \\
\hline 145 & $\begin{array}{l}\text { Acetes japonicus Kishinouye, } \\
1905\end{array}$ & $\begin{array}{l}\text { Southwest coast, Arabian Sea, } \\
\text { Bay of Bengal }\end{array}$ \\
\hline 146 & Acetes johni Nataraj, 1949 & Southwest coast \\
\hline 147 & $\begin{array}{l}\text { Acetes sibogae sibogae Hansen, } \\
1919\end{array}$ & West and southeast coast \\
\hline 148 & $\begin{array}{l}\text { Deosergestes rubroguttatus } \\
\text { (Wood-Mason in Wood-Mason \& } \\
\text { Alcock, 1891) }\end{array}$ & - \\
\hline 149 & $\begin{array}{l}\text { Deosergestes seminudus } \\
\text { (Hansen, 1919) }\end{array}$ & - \\
\hline 150 & $\begin{array}{l}\text { Neosergestes orientalis (Hansen, } \\
\text { 1919) }\end{array}$ & - \\
\hline 151 & $\begin{array}{l}\text { Neosergestes semissis } \\
\text { (Burkenroad, 1940) }\end{array}$ & - \\
\hline
\end{tabular}

\begin{tabular}{|c|c|c|}
\hline & Species name & Distribution \\
\hline 152 & $\begin{array}{l}\text { Parasergestes armatus (Krøyer, } \\
\text { 1855) }\end{array}$ & - \\
\hline 153 & $\begin{array}{l}\text { Sergestes hamifer Alcock \& } \\
\text { Anderson, } 1894\end{array}$ & $\begin{array}{l}\text { Arabian Sea, Andaman \& Nicobar } \\
\text { Islands }\end{array}$ \\
\hline 154 & $\begin{array}{l}\text { Sergia bisulcata (Wood-Mason \& } \\
\text { Alcock, 1891) }\end{array}$ & Arabian sea \\
\hline 155 & Sergia inoa (Faxon, 1893) & - \\
\hline \multicolumn{3}{|c|}{$\begin{array}{l}\text { Sub order: Pleocyemata Burkenroad, } 1963 \\
\text { Infra order: Axiidea de Saint Laurent, } 1979 \\
\text { Family: Axiidae Huxley, } 1879\end{array}$} \\
\hline 156 & $\begin{array}{l}\text { Ambiaxius alcocki (McArdle, } \\
1900 \text { ) }\end{array}$ & Southwest coast \\
\hline 157 & Calaxiopsis felix (Alcock, 1899) & - \\
\hline 158 & $\begin{array}{l}\text { Eiconaxius andamanensis } \\
\text { (Alcock, 1901) }\end{array}$ & Andaman \& Nicobar Islands \\
\hline 159 & $\begin{array}{l}\text { Eiconaxius laccadivensis Alcock \& } \\
\text { Anderson, } 1894\end{array}$ & Lakshadweep Islands \\
\hline \multicolumn{3}{|c|}{$\begin{array}{l}\text { Family: Callianassidae Dana, } 1852 \\
\text { Subfamily: Callianassinae Dana, } 1852\end{array}$} \\
\hline 160 & $\begin{array}{l}\text { Callianassa lignicola Alcock \& } \\
\text { Anderson, } 1899\end{array}$ & Bay of Bengal \\
\hline \multicolumn{3}{|c|}{$\begin{array}{l}\text { Infra order: Caridea Latreille, } 1817 \\
\text { Super family: Alpheoidea Rafinesque, } 1815 \\
\text { Family: Alpheidae Rafinesque, } 1815\end{array}$} \\
\hline 161 & $\begin{array}{l}\text { Alpheus acutofemoratus Dana, } \\
1852\end{array}$ & Andaman \& Nicobar Islands \\
\hline 162 & Alpheus armatus Rathbun, 1901 & Chennai \\
\hline 163 & $\begin{array}{l}\text { Alpheus crockeri (Armstrong, } \\
\text { 1941) }\end{array}$ & Andaman \& Nicobar Islands \\
\hline 164 & Alpheus digitalis De Haan, 1844 & Gulf of Kutch, Gujarat \\
\hline 165 & $\begin{array}{l}\text { Alpheus edwardsii (Audouin, } \\
\text { 1826) }\end{array}$ & West Bengal \\
\hline 166 & $\begin{array}{l}\text { Alpheus euphrosyne de Man, } \\
1897\end{array}$ & West Bengal and Tamil Nadu \\
\hline 167 & $\begin{array}{l}\text { Alpheus frontalis H. Milne } \\
\text { Edwards, } 1837\end{array}$ & Andaman \& Nicobar Island \\
\hline 168 & Alpheus lobidens De Haan, 1849 & - \\
\hline 169 & $\begin{array}{l}\text { Alpheus macroskeles Alcock \& } \\
\text { Anderson, } 1899\end{array}$ & Bay of Bengal \\
\hline 170 & $\begin{array}{l}\text { Alpheus malabaricus (Fabricius, } \\
1775 \text { ) }\end{array}$ & Kerala \&Southeast coast \\
\hline 171 & Alpheus miersi Coutière, 1898 & Lakshadweep Islands \\
\hline 172 & Alpheus paludicola Kemp, 1915 & Karnataka \\
\hline 173 & Alpheus rapax Fabricius, 1798 & Lakshadweep Islands \\
\hline 174 & $\begin{array}{l}\text { Alpheus spongiarum Coutière, } \\
1897\end{array}$ & Lakshadweep Islands \\
\hline 175 & $\begin{array}{l}\text { Alpheus strenuus strenuus Dana, } \\
1852\end{array}$ & Andaman \& Nicobar Islands \\
\hline 176 & Arete dorsalis Stimpson, 1860 & Andaman \& Nicobar Islands \\
\hline 177 & $\begin{array}{l}\text { Athanas areteformis Coutière, } \\
1903\end{array}$ & Lakshadweep Islands \\
\hline 178 & $\begin{array}{l}\text { Athanas dimorphus Ortmann, } \\
1894\end{array}$ & Karwar \\
\hline 179 & $\begin{array}{l}\text { Athanas djiboutensis Coutière, } \\
1897\end{array}$ & Lakshadweep Islands \\
\hline 180 & $\begin{array}{l}\text { Synalpheus herdmaniae Lebour, } \\
1938\end{array}$ & Gulf of Mannar \\
\hline 181 & $\begin{array}{l}\text { Synalpheus paraneomeris } \\
\text { Coutière, } 1905\end{array}$ & - \\
\hline 182 & $\begin{array}{l}\text { Synalpheus paulsoni } \\
\text { rameswarensis Coutière, } 1908\end{array}$ & Gulf of Mannar \\
\hline
\end{tabular}




\begin{tabular}{|c|c|c|}
\hline & Species name & Distribution \\
\hline 183 & $\begin{array}{l}\text { Synalpheus tumidomanus } \\
\text { tumidomanus (Paul'son, 1875) }\end{array}$ & Karwar \\
\hline \multicolumn{3}{|c|}{ Family: Hippolytidae Spence Bate, 1888} \\
\hline 184 & $\begin{array}{l}\text { Exhippolysmata ensirostris } \\
\text { ensirostris (Kemp, 1914) }\end{array}$ & East and West coast \\
\hline 185 & $\begin{array}{l}\text { Exhippolysmata punctata (Kemp, } \\
\text { 1914) }\end{array}$ & East and West coast \\
\hline 186 & Latreutes anoplonyx Kemp, 1914 & Northwest coast \\
\hline 187 & $\begin{array}{l}\text { Latreutes mucronatus (Stimpson, } \\
1860 \text { ) }\end{array}$ & Kerala \\
\hline 188 & Latreutes porcinus Kemp, 1916 & Andaman \& Nicobar Islands \\
\hline 189 & Latreutes pymoeus Nobili, 1904 & - \\
\hline 190 & $\begin{array}{l}\text { Lysmata amboinensis (de Man, } \\
\text { 1888) }\end{array}$ & - \\
\hline 191 & Lysmata vittata (Stimpson, 1860) & Maharashtra \\
\hline 192 & $\begin{array}{l}\text { Merhippolyte calmani Kemp \& } \\
\text { Sewell, } 1912\end{array}$ & Southwest coast \\
\hline 193 & $\begin{array}{l}\text { Paralatreutes bicornis Kemp, } \\
1925\end{array}$ & Andaman \& Nicobar Islands \\
\hline 194 & Phycocaris simulans Kemp, 1916 & Andaman \& Nicobar Islands \\
\hline 195 & Saron marmoratus (Olivier, 1811) & Gulf of Kutch, West Bengal \\
\hline 196 & Saron neglectus de Man, 1902 & Andaman \& Nicobar Islands \\
\hline 197 & $\begin{array}{l}\text { Thinora maldivensis Borradaile, } \\
1915\end{array}$ & Lakshadweep Islands \\
\hline 198 & Thor amboinensis (de Man, 1888) & Andaman \& Nicobar Islands \\
\hline 199 & Thor paschalis (Heller, 1862) & Andaman \& Nicobar Islands \\
\hline 200 & Tozeuma armatum Paulson, 1875 & Arabian sea \\
\hline \multicolumn{3}{|c|}{ Family: Ogyrididae Holthuis, 1955} \\
\hline 201 & $\begin{array}{l}\text { Ogyrides orientalis (Stimpson, } \\
1860 \text { ) }\end{array}$ & - \\
\hline 202 & $\begin{array}{l}\text { Ogyrides striaticauda Kemp, } \\
1915\end{array}$ & Chilka Lake, Odisha \\
\hline \multicolumn{3}{|c|}{$\begin{array}{l}\text { Super family: Bresilioidea Calman, } 1896 \\
\text { Family: Disciadidae Rathbun, } 1902\end{array}$} \\
\hline 203 & Discias exul Kemp, 1920 & - \\
\hline \multicolumn{3}{|c|}{$\begin{array}{l}\text { Super family: Crangonoidea Haworth, } 1825 \\
\text { Family: Crangonidae Haworth, } 1825\end{array}$} \\
\hline 204 & Aegaeon lacazei (Gourret, 1887) & Chennai \\
\hline 205 & $\begin{array}{l}\text { Parapontocaris andamanensis } \\
\text { (Wood-Mason in Wood-Mason \& } \\
\text { Alcock, 1891) }\end{array}$ & Andaman \& Nicobar Islands \\
\hline 206 & $\begin{array}{l}\text { Parapontocaris bengalensis } \\
\text { (Wood-Mason in Wood-Mason \& } \\
\text { Alcock, 1891) }\end{array}$ & Bay of Bengal \\
\hline 207 & $\begin{array}{l}\text { Parapontophilus abyssi (Smith, } \\
\text { 1884) }\end{array}$ & - \\
\hline 508 & $\begin{array}{l}\text { Parapontophilus gracilis gracilis } \\
\text { (Smith, 1882) }\end{array}$ & - \\
\hline 209 & $\begin{array}{l}\text { Philocheras angustirostris (de } \\
\text { Man, 1918) }\end{array}$ & Andaman \& Nicobar Islands \\
\hline 210 & $\begin{array}{l}\text { Philocheras candidus (Kemp, } \\
\text { 1916) }\end{array}$ & Andaman \& Nicobar Islands \\
\hline 211 & $\begin{array}{l}\text { Philocheras hendersoni (Kemp, } \\
\text { 1915) }\end{array}$ & Andaman \& Nicobar Islands \\
\hline 212 & Philocheras incisus (Kemp, 1916) & Andaman \& Nicobar Islands \\
\hline 213 & Philocheras lowisi (Kemp, 1916) & Andaman \& Nicobar Islands \\
\hline 214 & $\begin{array}{l}\text { Philocheras parvirostris (Kemp, } \\
\text { 1916) }\end{array}$ & Gulf of Mannar \\
\hline 215 & Philocheras pilosus (Kemp, 1916) & Gulf of Mannar \\
\hline
\end{tabular}

\begin{tabular}{|c|c|c|}
\hline & Species name & Distribution \\
\hline 216 & Philocheras plebs (Kemp, 1916) & Andaman \& Nicobar Islands \\
\hline 217 & $\begin{array}{l}\text { Philocheras sabsechota (Kemp, } \\
\text { 1911) }\end{array}$ & Andaman \& Nicobar Islands \\
\hline 218 & $\begin{array}{l}\text { Pontocaris affinis affinis (Alcock, } \\
\text { 1901) }\end{array}$ & Northwest coast \\
\hline 219 & $\begin{array}{l}\text { Pontocaris pennata (Spence Bate, } \\
\text { 1888) }\end{array}$ & Chennai, Kakinada \\
\hline 220 & $\begin{array}{l}\text { Pontocaris propensalata (Spence } \\
\text { Bate, 1888) }\end{array}$ & Andaman \& Nicobar Islands \\
\hline 221 & $\begin{array}{l}\text { Prionocrangon ommatosteres } \\
\text { Wood-Mason in Wood-Mason \& } \\
\text { Alcock, } 1891\end{array}$ & - \\
\hline \multicolumn{3}{|c|}{ Family: Glyphocrangonidae Smith, 1884} \\
\hline 222 & $\begin{array}{l}\text { Glyphocrangon andamanensis } \\
\text { Wood-Mason in Wood-Mason \& } \\
\text { Alcock, } 1891\end{array}$ & Andaman \& Nicobar Islands \\
\hline 223 & $\begin{array}{l}\text { Glyphocrangon caeca Wood- } \\
\text { Mason in Wood-Mason \& Alcock, } \\
1891\end{array}$ & Bay of Bengal \\
\hline 224 & $\begin{array}{l}\text { Glyphocrangon caecescens } \\
\text { Wood-Mason in Wood-Mason \& } \\
\text { Alcock, } 1891\end{array}$ & Bay of Bengal \\
\hline 225 & $\begin{array}{l}\text { Glyphocrangon cerea Alcock \& } \\
\text { Anderson, } 1894\end{array}$ & Lakshadweep Islands \\
\hline 226 & $\begin{array}{l}\text { Glyphocrangon gilesii Wood- } \\
\text { Mason \& Alcock, } 1891 \\
\end{array}$ & Andaman \& Nicobar Islands \\
\hline 227 & $\begin{array}{l}\text { Glyphocrangon investigatoris } \\
\text { Wood-Mason \& Alcock, } 1891\end{array}$ & Chennai \\
\hline 228 & $\begin{array}{l}\text { Glyphocrangon priononota } \\
\text { Wood-Mason \& Alcock, } 1891\end{array}$ & Lakshadweep Islands \\
\hline 229 & $\begin{array}{l}\text { Glyphocrangon regalis Spence } \\
\text { Bate, } 1888\end{array}$ & Kerala, Southwest coast \\
\hline 230 & $\begin{array}{l}\text { Glyphocrangon smithii Wood- } \\
\text { Mason in Wood-Mason \& Alcock, } \\
1891\end{array}$ & Andaman \& Nicobar Islands \\
\hline 231 & $\begin{array}{l}\text { Glyphocrangon unguiculata } \\
\text { Wood-Mason \& Alcock, } 1891\end{array}$ & Arabian sea \\
\hline \multicolumn{3}{|c|}{$\begin{array}{l}\text { Super family: Nematocarcinoidea Smith, } 1884 \\
\text { Family: Nematocarcinidae Smith, } 1884\end{array}$} \\
\hline 232 & $\begin{array}{l}\text { Nematocarcinus cursor A. Milne- } \\
\text { Edwards, } 1881\end{array}$ & - \\
\hline 233 & $\begin{array}{l}\text { Nematocarcinus tenuirostris } \\
\text { Spence Bate, } 1888\end{array}$ & $\begin{array}{l}\text { Arabian sea, Andaman \& Nicobar } \\
\text { Islands }\end{array}$ \\
\hline 234 & $\begin{array}{l}\text { Nematocarcinus undulatipes } \\
\text { Spence Bate, } 1888\end{array}$ & Lakshadweep Islands \\
\hline \multicolumn{3}{|c|}{ Family: Rhynchocinetidae Ortmann, 1890} \\
\hline 235 & $\begin{array}{l}\text { Cinetorhynchus hendersoni } \\
\text { (Kemp, 1925) }\end{array}$ & Andaman \& Nicobar Islands \\
\hline 236 & $\begin{array}{l}\text { Rhynchocinetes durbanensis } \\
\text { Gordon, } 1936\end{array}$ & Netrani Island, Karnataka \\
\hline \multicolumn{3}{|c|}{$\begin{array}{l}\text { Super family: Oplophoroidea Dana, } 1852 \\
\text { Family: Acanthephyridae Spence Bate, } 1888\end{array}$} \\
\hline 237 & $\begin{array}{l}\text { Acanthephyra armata A. Milne- } \\
\text { Edwards, } 1881\end{array}$ & - \\
\hline 238 & $\begin{array}{l}\text { Acanthephyra curtirostris Wood- } \\
\text { Mason \& Alcock, } 1891\end{array}$ & - \\
\hline 239 & Acanthephyra eximia Smith, 1884 & - \\
\hline 240 & $\begin{array}{l}\text { Acanthephyra fimbriata Alcock \& } \\
\text { Anderson, } 1894\end{array}$ & Bay of Bengal \\
\hline 241 & $\begin{array}{l}\text { Acanthephyra sanguinea Wood- } \\
\text { Mason [in Wood-Mason \& } \\
\text { Alcock, 1892] }\end{array}$ & Kerala, west coast \\
\hline 242 & $\begin{array}{l}\text { Ephyrina hoskynii Wood-Mason } \\
\text { \& Alcock, } 1891\end{array}$ & Bay of Bengal \\
\hline
\end{tabular}




\begin{tabular}{|c|c|c|}
\hline & Species name & Distribution \\
\hline 243 & $\begin{array}{l}\text { Heterogenys microphthalma } \\
\text { (Smith, 1885) }\end{array}$ & - \\
\hline 244 & Hymenodora gracilis Smith, 1886 & West coast \\
\hline 245 & Meningodora vesca (Smith, 1886) & Bay of Bengal \\
\hline 246 & $\begin{array}{l}\text { Notostomus sp. A. Milne- } \\
\text { Edwards, } 1881\end{array}$ & West coast \\
\hline \multicolumn{3}{|c|}{ Family: Oplophoridae Dana, 1852} \\
\hline 247 & $\begin{array}{l}\text { Oplophorus gracilirostris A. } \\
\text { Milne-Edwards, } 1881\end{array}$ & Southwest coast \\
\hline 248 & $\begin{array}{l}\text { Oplophorus spinosus (Brullé, } \\
\text { 1839) }\end{array}$ & Southwest coast \\
\hline 249 & $\begin{array}{l}\text { Oplophorus typus H. Milne } \\
\text { Edwards, } 1837 \text { [in H. Milne } \\
\text { Edwards, 1834-1840] }\end{array}$ & West coast \\
\hline \multicolumn{3}{|c|}{$\begin{array}{l}\text { Super family: Palaemonoidea Rafinesque, } 1815 \\
\text { Family: Gnathophyllidae Dana, } 1852\end{array}$} \\
\hline 250 & Pycnocaris chagoae Bruce, 1972 & Agatti, Lakshadweep Islands \\
\hline \multicolumn{3}{|c|}{ Family: Hymenoceridae Ortmann, 1890} \\
\hline 251 & Hymenocera picta Dana, 1852 & Minicoy \\
\hline \multicolumn{3}{|c|}{ Family: Palaemonidae Rafinesque, 1815} \\
\hline 252 & Leander tenuicornis (Say, 1818) & - \\
\hline 253 & Palaemon belindae (Kemp, 1925) & - \\
\hline 254 & Palaemon concinnus Dana, 1852 & - \\
\hline 255 & Palaemon debilis Dana, 1852 & Andaman \& Nicobar Islands \\
\hline 256 & $\begin{array}{l}\text { Palaemon pacificus (Stimpson, } \\
\text { 1860) }\end{array}$ & - \\
\hline 257 & $\begin{array}{l}\text { Palaemon semmelinkii (de Man, } \\
\text { 1881) }\end{array}$ & - \\
\hline 258 & $\begin{array}{l}\text { Palaemon serrifer (Stimpson, } \\
\text { 1860) }\end{array}$ & - \\
\hline 259 & Palaemon sewelli (Kemp, 1925) & - \\
\hline \multicolumn{3}{|c|}{ Sub family: Palaemoninae Rafinesque, 1815} \\
\hline 260 & $\begin{array}{l}\text { Exopalaemon styliferus (H. Milne } \\
\text { Edwards, 1840) }\end{array}$ & - \\
\hline 261 & $\begin{array}{l}\text { Leandrites celebensis (de Man, } \\
\text { 1881) }\end{array}$ & Kerala, Tamil Nadu \\
\hline 262 & $\begin{array}{l}\text { Macrobrachium divakarani } \\
\text { Jayachandran, } 2001\end{array}$ & Kerala \\
\hline 263 & $\begin{array}{l}\text { Macrobrachium equidens (Dana, } \\
\text { 1852) }\end{array}$ & West Bengal, Kerala, Goa \\
\hline 264 & $\begin{array}{l}\text { Macrobrachium rosenbergii (de } \\
\text { Man, 1879) }\end{array}$ & $\begin{array}{l}\text { Kerala, Karnataka, West Bengal, } \\
\text { Andhra Pradesh, Maharashtra, } \\
\text { Tamil Nadu }\end{array}$ \\
\hline 265 & $\begin{array}{l}\text { Nematopalaemon karnafuliensis } \\
\text { (Ali Azam Khan, Fincham \& } \\
\text { Mahmood, 1980) }\end{array}$ & West Bengal \\
\hline 266 & $\begin{array}{l}\text { Nematopalaemon tenuipes } \\
\text { (Henderson, 1893) }\end{array}$ & $\begin{array}{l}\text { Northwest, south and northeast } \\
\text { coast }\end{array}$ \\
\hline 267 & $\begin{array}{l}\text { Rhopalaemon belindae (Kemp, } \\
\text { 1925) }\end{array}$ & Gulf of Mannar \\
\hline 268 & $\begin{array}{l}\text { Urocaridella urocaridella } \\
\text { (Holthuis, 1950) }\end{array}$ & Odisha \\
\hline \multicolumn{3}{|c|}{ Sub family: Pontoniinae Kingsley, 1879} \\
\hline 269 & $\begin{array}{l}\text { Allopontonia brockii (de Man, } \\
\text { 1888) }\end{array}$ & Andaman \& Nicobar Islands \\
\hline 270 & Anchistus custos (Forskål, 1775) & Andaman \& Nicobar Islands \\
\hline 271 & Anchistus demani Kemp, 1922 & Andaman \& Nicobar Islands \\
\hline 272 & Anchistus miersi (de Man, 1888) & Andaman \& Nicobar Islands \\
\hline 273 & Anchistus pectinis Kemp, 1925 & Andaman \& Nicobar Islands \\
\hline
\end{tabular}

\begin{tabular}{|c|c|c|}
\hline & Species name & Distribution \\
\hline 274 & $\begin{array}{l}\text { Conchodytes biunguiculatus } \\
\text { (Paul'son, 1875) }\end{array}$ & Andaman \& Nicobar Islands \\
\hline 275 & $\begin{array}{l}\text { Conchodytes placunae (Johnson, } \\
\text { 1967) }\end{array}$ & $\begin{array}{l}\text { Andaman \& Nicobar Islands, } \\
\text { southern India }\end{array}$ \\
\hline 276 & $\begin{array}{l}\text { Conchodytes tridacnae Peters, } \\
1852\end{array}$ & Andaman \& Nicobar Islands \\
\hline 277 & $\begin{array}{l}\text { Coralliocaris graminea (Dana, } \\
1852 \text { ) }\end{array}$ & Andaman \& Nicobar Islands \\
\hline 278 & $\begin{array}{l}\text { Coralliocaris nudirostris (Heller, } \\
\text { 1861) }\end{array}$ & Gulf of Mannar \\
\hline 279 & $\begin{array}{l}\text { Coralliocaris superba (Dana, } \\
\text { 1852) }\end{array}$ & Andaman \& Nicobar Islands \\
\hline 280 & Cuapetes agag (Kemp, 1922) & Andaman \& Nicobar Islands \\
\hline 281 & $\begin{array}{l}\text { Cuapetes amymone (de Man, } \\
\text { 1902) }\end{array}$ & Andaman \& Nicobar Islands \\
\hline 282 & $\begin{array}{l}\text { Cuapetes andamanensis (Kemp, } \\
\text { 1922) }\end{array}$ & Andaman \& Nicobar Islands \\
\hline 283 & Cuapetes demani (Kemp, 1915) & Chennai, Tamil Nadu \\
\hline 284 & $\begin{array}{l}\text { Cuapetes elegans (Paul'son, } \\
1875 \text { ) }\end{array}$ & Lakshadweep Islands \\
\hline 285 & $\begin{array}{l}\text { Cuapetes grandis (Stimpson, } \\
\text { 1860) }\end{array}$ & Kochi Backwaters \\
\hline 286 & $\begin{array}{l}\text { Cuapetes longirostris (Borradaile, } \\
\text { 1915) }\end{array}$ & Andaman \& Nicobar Islands \\
\hline 287 & $\begin{array}{l}\text { Cuapetes seychellensis } \\
\text { (Borradaile, 1915) }\end{array}$ & Andaman \& Nicobar Islands \\
\hline 288 & $\begin{array}{l}\text { Cuapetes tenuipes (Borradaile, } \\
\text { 1898) }\end{array}$ & Andaman \& Nicobar Islands \\
\hline 289 & $\begin{array}{l}\text { Dactylonia anachoreta (Kemp, } \\
\text { 1922) }\end{array}$ & Chennai, Tamil Nadu \\
\hline 290 & $\begin{array}{l}\text { Dasella herdmaniae (Lebour, } \\
\text { 1938) }\end{array}$ & Gulf of Mannar \\
\hline 291 & Dasycaris symbiotes Kemp, 1922 & Visakhapatnam \\
\hline 292 & $\begin{array}{l}\text { Harpiliopsis beaupresii (Audouin, } \\
\text { 1826) }\end{array}$ & Andaman \& Nicobar Islands \\
\hline 293 & $\begin{array}{l}\text { Harpiliopsis depressa (Stimpson, } \\
\text { 1860) }\end{array}$ & Tamil Nadu coast \\
\hline 294 & $\begin{array}{l}\text { Harpiliopsis spinigera (Ortmann, } \\
\text { 1890) }\end{array}$ & Andaman \& Nicobar Islands \\
\hline 295 & Jocaste lucina (Nobili, 1901) & Andaman \& Nicobar Islands \\
\hline 296 & Palaemonella lata Kemp, 1922 & Andaman \& Nicobar Islands \\
\hline 297 & $\begin{array}{l}\text { Palaemonella rotumana } \\
\text { (Borradaile, 1898) }\end{array}$ & Andaman \& Nicobar Islands \\
\hline 298 & $\begin{array}{l}\text { Periclimenella spinifera (de Man, } \\
\text { 1902) }\end{array}$ & Andaman \& Nicobar Islands \\
\hline 299 & $\begin{array}{l}\text { Periclimenes alcocki (Kemp, } \\
\text { 1922) }\end{array}$ & Andaman \& Nicobar Islands \\
\hline 300 & $\begin{array}{l}\text { Periclimenes brevicarpalis } \\
\text { (Schenkel, 1902) }\end{array}$ & Gulf of Mannar \\
\hline 301 & Periclimenes digitalis Kemp, 1922 & Andaman \& Nicobar Islands \\
\hline 302 & $\begin{array}{l}\text { Periclimenes diversipes Kemp, } \\
1922\end{array}$ & Gulf of Mannar \\
\hline 303 & $\begin{array}{l}\text { Periclimenes incertus Borradaile, } \\
1915\end{array}$ & Andaman \& Nicobar Islands \\
\hline 304 & $\begin{array}{l}\text { Periclimenes inornatus Kemp, } \\
1922\end{array}$ & Andaman \& Nicobar Islands \\
\hline 305 & Periclimenes kempi Bruce, 1969 & Andaman \& Nicobar Islands \\
\hline 306 & $\begin{array}{l}\text { Periclimenes laccadivensis } \\
\text { (Alcock \& Anderson, 1894) }\end{array}$ & Lakshadweep Islands \\
\hline 307 & $\begin{array}{l}\text { Periclimenes leptopus Kemp, } \\
1922\end{array}$ & Andaman \& Nicobar Islands \\
\hline 308 & $\begin{array}{l}\text { Periclimenes obscurus Kemp, } \\
1922\end{array}$ & Kerala, Trivandrum \\
\hline
\end{tabular}




\begin{tabular}{|c|c|c|}
\hline & Species name & Distribution \\
\hline 309 & $\begin{array}{l}\text { Periclimenes proximus Kemp, } \\
1922\end{array}$ & - \\
\hline 310 & Periclimenes rex Kemp, 1922 & Gulf of Mannar, Chennai \\
\hline 311 & Philarius gerlachei (Nobili, 1905) & Gulf of Mannar \\
\hline 312 & $\begin{array}{l}\text { Phycomenes indicus (Kemp, } \\
\text { 1915) }\end{array}$ & $\begin{array}{l}\text { South coast, Nicobar Island, } \\
\text { Chilka lake }\end{array}$ \\
\hline 313 & Vir orientalis (Dana, 1852) & Andaman \& Nicobar Islands \\
\hline \multicolumn{3}{|c|}{$\begin{array}{l}\text { Super family: Pandaloidea Haworth, } 1825 \\
\text { Family: Pandalidae Haworth, } 1825\end{array}$} \\
\hline 314 & $\begin{array}{l}\text { Chlorocurtis jactans (Nobili, } \\
\text { 1904) }\end{array}$ & Andaman \& Nicobar Islands \\
\hline 315 & Chlorotocella gracilis Balss, 1914 & Andaman \& Nicobar Islands \\
\hline 316 & $\begin{array}{l}\text { Chlorotocus crassicornis (A. } \\
\text { Costa, 1871) }\end{array}$ & Andaman \& Nicobar Islands \\
\hline 317 & $\begin{array}{l}\text { Dorodotes reflexus Spence Bate, } \\
1888\end{array}$ & Bay of Bengal \\
\hline 318 & $\begin{array}{l}\text { Heterocarpus dorsalis Spence } \\
\text { Bate, } 1888 \\
\end{array}$ & $\begin{array}{l}\text { Arabian Sea, Andaman \& Nicobar } \\
\text { Islands, Bay of Bengal }\end{array}$ \\
\hline 319 & $\begin{array}{l}\text { Heterocarpus ensifer A. Milne- } \\
\text { Edwards, } 1881\end{array}$ & Southeast and west coast \\
\hline 320 & $\begin{array}{l}\text { Heterocarpus gibbosus Spence } \\
\text { Bate, } 1888\end{array}$ & $\begin{array}{l}\text { Southeast and west coast, } \\
\text { Andaman \& Nicobar Islands }\end{array}$ \\
\hline 321 & \begin{tabular}{|l} 
Heterocarpus laevigatus Spence \\
Bate, 1888
\end{tabular} & Arabian Sea, Bay of Bengal \\
\hline 322 & $\begin{array}{l}\text { Heterocarpus longirostris } \\
\text { MacGilchrist, } 1905 \\
\end{array}$ & $\begin{array}{l}\text { Bay of Bengal, Andaman \& } \\
\text { Nicobar Islands }\end{array}$ \\
\hline 323 & \begin{tabular}{|l} 
Heterocarpus sibogae de Man, \\
1917
\end{tabular} & $\begin{array}{l}\text { Southwest coast, Andaman \& } \\
\text { Nicobar Islands } \\
\end{array}$ \\
\hline 324 & $\begin{array}{l}\text { Heterocarpus tricarinatus Alcock } \\
\text { \& Anderson, } 1894\end{array}$ & $\begin{array}{l}\text { Arabian Sea, Lakshadweep } \\
\text { Islands, Andaman \& Nicobar } \\
\text { Island }\end{array}$ \\
\hline 325 & $\begin{array}{l}\text { Heterocarpus woodmasoni } \\
\text { Alcock, } 1901\end{array}$ & $\begin{array}{l}\text { Southeast, west coast, } \\
\text { Andaman \& Nicobar Island }\end{array}$ \\
\hline 326 & $\begin{array}{l}\text { Parapandalus longicauda } \\
\text { (Rathbun, 1901) }\end{array}$ & Kakinada \\
\hline 327 & $\begin{array}{l}\text { Plesionika adensamer (Balss, } \\
\text { 1914) }\end{array}$ & Arabian Sea, Bay of Bengal \\
\hline 328 & $\begin{array}{l}\text { Plesionika alcocki (Anderson, } \\
\text { 1896) }\end{array}$ & Arabian Sea and Bay of Bengal \\
\hline 329 & $\begin{array}{l}\text { Plesionika bifurca Alcock \& } \\
\text { Anderson, } 1894\end{array}$ & $\begin{array}{l}\text { Andaman \& Nicobar Islands, } \\
\text { Arabian sea, Bay of Bengal }\end{array}$ \\
\hline 330 & $\begin{array}{l}\text { Plesionika ensis (A. Milne- } \\
\text { Edwards, 1881) }\end{array}$ & Southwest and East coast \\
\hline 331 & $\begin{array}{l}\text { Plesionika longicauda (Rathbun, } \\
\text { 1901) }\end{array}$ & Bay of Bengal \\
\hline 332 & $\begin{array}{l}\text { Plesionika martia A. Milne- } \\
\text { Edwards, } 1883\end{array}$ & $\begin{array}{l}\text { Andaman \& Nicobar Islands, } \\
\text { Arabian sea, Bay of Bengal }\end{array}$ \\
\hline 333 & $\begin{array}{l}\text { Plesionika ocellus (Spence Bate, } \\
\text { 1888) }\end{array}$ & $\begin{array}{l}\text { Andaman \& Nicobar Islands, } \\
\text { Arabian sea }\end{array}$ \\
\hline 334 & $\begin{array}{l}\text { Plesionika quasigrandis Chace, } \\
1985\end{array}$ & West and southeast coast \\
\hline 335 & Plesionika sindoi (Rathbun, 1906) & $\begin{array}{l}\text { Andaman \& Nicobar Islands, } \\
\text { Arabian Sea }\end{array}$ \\
\hline 336 & $\begin{array}{l}\text { Plesionika spinipes Spence Bate, } \\
1888\end{array}$ & $\begin{array}{l}\text { Arabian Sea, Andaman \& Nicobar } \\
\text { Islands, Bay of Bengal }\end{array}$ \\
\hline 337 & $\begin{array}{l}\text { Plesionika unidens Spence Bate, } \\
1888\end{array}$ & Andaman \& Nicobar Islands \\
\hline 338 & Plesionika williamsi Forest, 1964 & Southwest coast \\
\hline 339 & $\begin{array}{l}\text { Procletes levicarina (Spence Bate, } \\
1888 \text { ) }\end{array}$ & West coast \\
\hline
\end{tabular}

\begin{tabular}{|c|c|c|}
\hline & Species name & Distribution \\
\hline 340 & $\begin{array}{l}\text { Stylopandalus richardi (Coutière, } \\
\text { 1905) }\end{array}$ & Bay of Bengal \\
\hline \multicolumn{3}{|c|}{ Family: Thalassocarididae Spence Bate, 1888} \\
\hline 341 & $\begin{array}{l}\text { Chlorotocoides spinicauda (de } \\
\text { Man, 1902) }\end{array}$ & Andaman \& Nicobarlslands \\
\hline 342 & Thalassocaris crinita (Dana, 1852) & Andaman \& Nicobar Islands \\
\hline 343 & Thalassocaris lucida (Dana, 1852) & Southwest coast \\
\hline 344 & $\begin{array}{l}\text { Thalassocaris obscura (Gopala } \\
\text { Menon \& Williamson, 1971) }\end{array}$ & Arabian sea \\
\hline \multicolumn{3}{|c|}{$\begin{array}{l}\text { Super family: Pasiphaeoidea Dana, } 1852 \\
\text { Family: Pasiphaeidae Dana, } 1852\end{array}$} \\
\hline 345 & $\begin{array}{l}\text { Eupasiphae gilesii (Wood-Mason, } \\
\text { 1892) }\end{array}$ & Andaman \& Nicobar Islands \\
\hline 346 & $\begin{array}{l}\text { Eupasiphae latirostris (Wood- } \\
\text { Mason \& Alcock, 1891) }\end{array}$ & Arabian sea \\
\hline 347 & Glyphus marsupialis Filhol, 1884 & Arabian sea \\
\hline 348 & $\begin{array}{l}\text { Leptochela (Leptochela) } \\
\text { aculeocaudata Paul'son, } 1875\end{array}$ & Southwest coast \\
\hline 349 & $\begin{array}{l}\text { Leptochela (Leptochela) robusta } \\
\text { Stimpson, } 1860\end{array}$ & Southwest coast \\
\hline 350 & $\begin{array}{l}\text { Pasiphaea alcocki Wood-Mason } \\
\text { \& Alcock, } 1891\end{array}$ & Southwest, southeast coast \\
\hline 351 & $\begin{array}{l}\text { Pasiphaea unispinosa Wood- } \\
\text { Mason, } 1892\end{array}$ & Andaman \& Nicobar Islands \\
\hline 352 & $\begin{array}{l}\text { Psathyrocaris fragilis Wood- } \\
\text { Mason in Wood-Mason \& Alcock, } \\
1893\end{array}$ & - \\
\hline 353 & $\begin{array}{l}\text { Psathyrocaris infirma Alcock \& } \\
\text { Anderson, } 1894\end{array}$ & Southwest coast \\
\hline 354 & $\begin{array}{l}\text { Psathyrocaris platyophthalmus } \\
\text { Alcock \& Anderson, } 1894\end{array}$ & Lakshadweep Islands \\
\hline 355 & $\begin{array}{l}\text { Psathyrocaris plumosa Alcock \& } \\
\text { Anderson, } 1894\end{array}$ & - \\
\hline \multicolumn{3}{|c|}{$\begin{array}{l}\text { Super family: Processoidea Ortmann, } 1896 \\
\text { Family: Processidae Ortmann, } 1896\end{array}$} \\
\hline 356 & $\begin{array}{l}\text { Hayashidonus japonicus (De } \\
\text { Haan, 1844) }\end{array}$ & - \\
\hline 357 & Nikoides danae Paul'son, 1875 & Andaman \& Nicobar Islands \\
\hline 358 & Nikoides sibogae de Man, 1918 & - \\
\hline \multicolumn{3}{|c|}{$\begin{array}{l}\text { Super family: Psalidopodoidea Wood-Mason [in Wood-Mason \& Alcock } \\
\text { 1892] } \\
\text { Family: Psalidopodidae Wood-Mason [in Wood-Mason \& Alcock, 1892] }\end{array}$} \\
\hline 359 & \begin{tabular}{|l|} 
Psalidopus huxleyi Wood-Mason \\
[in Wood-Mason \& Alcock, 1892]
\end{tabular} & Andaman \& Nicobar Islands \\
\hline \multicolumn{3}{|c|}{$\begin{array}{l}\text { Infra order: Stenopodidea Spence Bate, } 1888 \\
\text { Family: Spongicolidae Schram, } 1986\end{array}$} \\
\hline 360 & $\begin{array}{l}\text { Engystenopus palmipes Alcock \& } \\
\text { Anderson, } 1894\end{array}$ & - \\
\hline 361 & $\begin{array}{l}\text { Microprosthema validum } \\
\text { Stimpson, } 1860\end{array}$ & Gulf of Mannar \\
\hline 362 & $\begin{array}{l}\text { Spongicola andamanicus Alcock, } \\
1901\end{array}$ & Andaman \& Nicobar Islands \\
\hline \multicolumn{3}{|c|}{ Family: Stenopodidae Claus, 1872} \\
\hline 363 & $\begin{array}{l}\text { Odontozona spongicola (Alcock \& } \\
\text { Anderson, 1899) }\end{array}$ & - \\
\hline 364 & Stenopus hispidus (Olivier, 1811) & Lakshadweep Islands \\
\hline
\end{tabular}


Oploporidae, but in the present list the genus is placed in the family Acanthephyridae (Fransen 2015e). The work carried out by Radhakrishnan et al. (2012) reports 343 species of marine shrimps containing 322 accepted names and 21 species names with taxonomic issues. These issues were 15 synonyms, subsequently updated with accepted names and six species names were dropped from the list. Further, 16 species not included in the list by Radhakrishnan et al. (2012) were added, of which nine species were from the work of Mohamed (1969), one species from Devi (1980), one species from Kurup et al. (2008), and five species from Shanis et al. (2012). Works published later by Chakraborthy (2013) and Rajakumaran \& Vaseeharan (2014) further added $11(1+10)$ more species to the checklist (Table. 3$)$.

A total of 27 species has been added to the checklist published in 2012 by Radhakrishnan et al. Altogether 25 issues were identified (all synonyms), out of which 19 species have been updated with the accepted names (Table 2) and six species dropped. After validation, 364 species have been reported in the present checklist (Table 1). Synonyms contributed to $5.2 \%$ of species names and the list of synonyms used by various authors is portrayed in Table 2. The work of Radhakrishnan et al. (2012) contained 21 issues whereas two issues were identified in Kasim (1969), namely Parapenaeopsis hungerfoldii (Alcockpaneopsis hungerfoldii - accepted name); Palaemon styliferus (Exopalaemon styliferus - accepted name). Two additional issues were identified in the work of Rajakumaran \& Vaseeharan (2014): Parapenaeposis sinica (Kishinouyepenaeopsis amicus - accepted name); Parapenaeopsis venusta (Batepenaeopsis venusta accepted name). Table 3 provides information on the number of species documented in each report, issues in each checklist, numbers of invalid species names that were dropped and revised and accepted names. The
Table 2. Synonyms of accepted shrimp species recorded from Indian waters

\begin{tabular}{|c|c|c|}
\hline Sno & Current name & Synonymised name \\
\hline 1 & $\begin{array}{l}\text { Penaeus konkani (Chanda \& } \\
\text { Bhattacharya, 2003) }\end{array}$ & $\begin{array}{l}\text { Fenneropenaeus konkani } \\
\text { Chanda \& Bhattacharya, } 2003\end{array}$ \\
\hline 2 & $\begin{array}{l}\text { Penaeus silasi Muthu \& Motoh, } \\
1979\end{array}$ & $\begin{array}{l}\text { Fenneropenaeus silasi (Muthu \& } \\
\text { Motoh, 1979) }\end{array}$ \\
\hline 3 & Penaeus vannamei Boone, 1931 & $\begin{array}{l}\text { Litopenaeus vannamei (Boone, } \\
\text { 1931) }\end{array}$ \\
\hline 4 & $\begin{array}{l}\text { Penaeus hathor (Burkenroad, } \\
\text { 1959) }\end{array}$ & $\begin{array}{l}\text { Melicertus hathor (Burkenroad, } \\
\text { 1959) }\end{array}$ \\
\hline 5 & $\begin{array}{l}\text { Penaeus similis (Chanda \& } \\
\text { Bhattacharya, 2002) }\end{array}$ & $\begin{array}{l}\text { Melicertus similis Chanda \& } \\
\text { Bhattacharya, } 2002\end{array}$ \\
\hline 6 & $\begin{array}{l}\text { Alcockpenaeopsis hungerfordii } \\
\text { (Alcock, 1905) }\end{array}$ & $\begin{array}{l}\text { Parapenaeopsis hungerfordii } \\
\text { Alcock, } 1905\end{array}$ \\
\hline 7 & $\begin{array}{l}\text { Kishinouyepenaeopsis amicus } \\
\text { (V.C. Nguyên, 1971) }\end{array}$ & $\begin{array}{l}\text { Parapenaeopsis sinica Liu \& } \\
\text { Wang, } 1986\end{array}$ \\
\hline 8 & $\begin{array}{l}\text { Batepenaeopsis venusta (de } \\
\text { Man, 1907) }\end{array}$ & $\begin{array}{l}\text { Parapeneopsis venusta de Man, } \\
1907\end{array}$ \\
\hline 9 & $\begin{array}{l}\text { Cerataspis monstruosus Gray, } \\
1828\end{array}$ & $\begin{array}{l}\text { Plesiopenaeus armatus (Spence } \\
\text { Bate, 1881) }\end{array}$ \\
\hline 10 & $\begin{array}{l}\text { Cerataspis coruscans (Wood- } \\
\text { Mason in Wood-Mason \& } \\
\text { Alcock, 1891) }\end{array}$ & $\begin{array}{l}\text { Plesiopenaeus coruscans } \\
\text { (Wood-Mason in Wood-Mason } \\
\text { \& Alcock, 1891) }\end{array}$ \\
\hline 11 & $\begin{array}{l}\text { Bentheogennema pasithea (de } \\
\text { Man, 1907) }\end{array}$ & Gennadas praecox Kemp, 1910 \\
\hline 12 & $\begin{array}{l}\text { Alpheus strenuus strenuous } \\
\text { Dana, } 1852\end{array}$ & Alpheus strenuous (Dana, 1852) \\
\hline 13 & $\begin{array}{l}\text { Synalpheus tumidomanus } \\
\text { tumidomanus (Paul'son, 1875) }\end{array}$ & $\begin{array}{l}\text { Synalpheus tumidomanus } \\
\text { (Paul'son, 1875) }\end{array}$ \\
\hline 14 & $\begin{array}{l}\text { Thinora maldivensis Borradaile, } \\
1915\end{array}$ & $\begin{array}{l}\text { Thor maldivensis Borradaile, } \\
1915\end{array}$ \\
\hline 15 & $\begin{array}{l}\text { Exopalaemon styliferus ( } \mathrm{H} . \\
\text { Milne Edwards, 1840) }\end{array}$ & $\begin{array}{l}\text { Palaemon styliferus H. Milne } \\
\text { Edwards, } 1840\end{array}$ \\
\hline 16 & $\begin{array}{l}\text { Conchodytes placunae (Johnson, } \\
1967 \text { ) }\end{array}$ & $\begin{array}{l}\text { Chernocaris placunae Johnson, } \\
1967\end{array}$ \\
\hline 17 & $\begin{array}{l}\text { Conchodytes biunguiculatus } \\
\text { (Paul'son, 1875) }\end{array}$ & Conchodytes kempi Bruce, 1989 \\
\hline 18 & $\begin{array}{l}\text { Coralliocaris nudirostris (Heller, } \\
\text { 1861) }\end{array}$ & $\begin{array}{l}\text { Coralliocaris venusta Kemp, } \\
1922\end{array}$ \\
\hline 19 & $\begin{array}{l}\text { Chlorotocoides spinicauda (de } \\
\text { Man, 1902) }\end{array}$ & $\begin{array}{l}\text { Chlorotocus spinicauda de Man, } \\
1902\end{array}$ \\
\hline
\end{tabular}

Table 3. Identified issues and revisions of shrimp species

\begin{tabular}{|c|c|c|c|c|c|c|}
\hline \multirow{2}{*}{ Authors } & \multicolumn{2}{|c|}{ Species number } & \multirow{2}{*}{$\begin{array}{c}\text { Issues } \\
\text { (synonyms) }\end{array}$} & \multirow{2}{*}{$\begin{array}{l}\text { Revised \& } \\
\text { Accepted }\end{array}$} & \multirow[b]{2}{*}{ Dropped } & \multirow[b]{2}{*}{ Final Checklist } \\
\hline & $\begin{array}{c}\text { Total } \\
\text { Reported }\end{array}$ & Accepted & & & & \\
\hline Radhakrishnan et al. 2012 & 437 & 343 & 21 & 15 & 6 & 337 \\
\hline Kasim 1969 & 93 & 2 & 2 & 2 & - & 9 \\
\hline Devi 1980 & 3 & 1 & - & - & - & 1 \\
\hline Kurup et al. 2008 & 37 & 1 & - & - & - & 1 \\
\hline Shanis et al. 2012 & 24 & 5 & - & & - & 5 \\
\hline Chakraborthy 2013 & 22 & 1 & - & - & - & 1 \\
\hline Rajakumaran \& Vaseeharan 2014 & 59 & 11 & 2 & 2 & - & 10 \\
\hline Total & -- & 364 & 25 & & 6 & 364 \\
\hline
\end{tabular}


total numbers contributed finally after the process of taxonomic validation resulted in 364 species from Indian waters.

The constant updating of national and regional level species checklists is to suit the objective proposed by Global Biodiversity Information Facility (GBIF) "to coordinate, consolidate and disseminate basic taxonomic and species information that is commonly required by a range of users". The updated checklist for shrimps in India in this report is a product of rendering services for biodiversity, conservation and management approaches.

\section{REFERENCES}

Alcock, A. \& A.R. Anderson (1894). Natural history notes from H.M. Indian Marine Survey Ship "Investigator". Series II, No. 14. An account of a recent collection of deep-sea Crustacea from the Bay of Bengal and Laccadive Sea. The Journal of the Asiatic Society of Bengal 63: 141-185.

Alcock, A \& A.R. Anderson (1899). Natural History Notes from H.M. Royal Indian Marine Survey Ship "Investigator," series III, No.22: An Account of the Deep-Sea Crustacea, dredged during the Surveying Season of 1897-98. Annals \& Magazine of Natural History 3: 278292.

Alcock, A. (1901). A descriptive catalogue of Indian deep-sea Crustacea, Decapoda, Macrura and Anomala in the Indian Museum. Baptist Mission Press, Calcutta, India, 286pp.

Abello, P., F.J. Valladares \& A. Castellon (1988). Analysis of the structure of crustacean assemblages off the Catlan coast (northwest Mediterranean). Marine Biology 98: 39-49.

Anon (2014). Handbook on Fisheries Statistics. Fishery Survey of India, Mumbai, $166 \mathrm{pp}$.

Chakraborthy, R.D. (2013). Deep sea prawns, pp. 107-131. In: Jose, J. \& L. Pillai (eds.). Training Programme on 'Taxonomy and identification of commercially important crustaceans of India'. Central Marine Fisheries Research Institute Publication, Kochi, India.

DAHDF (2014). Annual Report 2013-14. Department of Animal Husbandry, Dairying and Fisheries, Ministry of Agriculture Government of India, 130pp.

De Grave, S. (2015). Thinora maldivensis Borradaile, 1915 Accessed through: World Register of Marine Species at http:// www. marineSpecies.org/aphia. php?p=taxdetails \&id $=515341$. Downloaded on 04 August 2015.

Devi, L.S. (1980). Notes on three caridean prawns from Kakinada. Journal of the Marine Biological Association of India 22(1\&2): 169173.

Fransen, C. \& S. De Grave (2015). Penaeus. Accessed through: World Register of Marine Species at http://marineSpecies.org/aphia. php?p=taxdetails\&id=106822. Downloaded on 11 March 2015

Fransen, C. (2015a). Alcockpenaeopsis hungerfordii (Alcock, 1905). Accessed through: World Register of Marine Species at http:// www. marineSpecies.org/aphia.php?p=taxdetails \&id $=584932$. Downloaded on 11 March 2015.

Fransen, C. (2015b). Batepenaeopsis venusta (de Man, 1907). Accessed through: World Register of Marine Species at http:// www. marineSpecies.org/aphia.php? $p=$ taxdetails\&id=584940. Downloaded on 11 March 2015.

Fransen, C. (2015c). Cerataspis. Accessed through: World Register of Marine Species at http://www.marineSpecies.org/aphia. php?p=taxdetails\&id=589793. Downloaded on 11 March 2015.

Fransen, C. (2015d). Hippolytidae Spence Bate, 1888. Accessed through: World Register of Marine Species at http://www. marinespecies.org/aphia.php?p=taxdetails\&id=106777. Downloaded on 10 April 2015.

Fransen, C. (2015e). Acanthephyridae Spence Bate, 1888. Accessed through: World Register of Marine Species at http:// www. marinespecies.org/aphia. php p $=$ taxdetails \&id $=585943$. Downloaded on 14 August 2015.

George, M.J. (1966). On a collection of the penaeid shrimp from the offshore waters off the south-west coast of India. Proceedings of the Symposium on Crustacea. Journal of the Marine Biological Association of India 1: 337-346.

Jayachandran, K.V. (2005). The biodiversity of palaemonid prawns from Indian Seas (in Hindi), pp. 21-28. In: Aspects of Aquatic Biodiversity. Central Marine Fisheries Research Institute, Cochin.

Jayachandran, K.V. (2010). Indian palaemonid decapod crustaceans: taxonomic status, research challenges and conservation needs. The Indian Journal of Animal Sciences 80(4)13: 46-52.

Karuppasamy, P.K., N.G. Menon, K.K.C. Nair \& C.T. Achuthankutty (2006). Distribution and abundance of pelagic shrimps from the deep scattering layer of the eastern Arabian Sea. Journal of Shellfish Research 25(3): 1013-1019.

Kasim, M.H. (1969). Catalogue of Prawns in the Reference Collections of the Central Marine Fisheries Reseach Institute, Catalogue of Molluscs, Prawns, Stomatopods and Marine Algae in the Reference Collections of the Central Marine Fisheries Research Institute, CMFRI Bulletin 9: 27-34.

Kathirvel, M., P. Thirumilu \& A. Gokul (2007). Indian penaeid shrimps - their biodiversity and economical values, pp. 161-176. In: National symposium on Conservation and Valuation of Marine Biodiversity. Kolkata, Zoological Survey of India.

Kemp, S. (1925). Notes on Crustacea Decapoda in the Indian Museum, XVII: On various Caridea. Records of Indian Museum 27(4): 249-344.

Kunju, M.M. (1960). On a new records of five Species of Penaeidae (Decapoda: Macrura Penaeidae) on the West Coast of India. Journal of the Marine Biological Association of India 2(1): 82-84.

Kurup, M.B., R. Rajasree \& S. Venu (2008). Distribution of deep sea prawns off Kerala. Journal of the Marine Biological Association of India 50(2): 122-126; http://dx.doi.org/10.6024/ jmbai.2012.54.1.01690-07

Mohamed, K.H. \& C. Suseelan (1973). Deep-sea prawn resources off the South-West Coast of India. Proceeding of the Symposium on Living Resources of the Seas around India, 614-633pp.

Muthu, M.S. \& M.J. George (1971). Solenocera indica Nataraj, one of the commercially important penaeid prawns of Indian waters as a synonym of Solenocera crassicornis ( $\mathrm{H}$. Milne Edwards). Journal of the Marine Biological Association of India 13: 142-143.

Prakash, S., I. Babu, M. Gopi, T.T. Ajithkumar \& T. Balasubramanyam (2011). Discovery of the shrimp Pycnocaris chagoae Bruce, 1972 (Decapoda: Caridea: Gnathophyllidae) in the Lakshadweep Archipelago. Zootaxa 2988: 66-68; http://dx.doi.org/10.11646/ zootaxa.3914.4.5

Radhakrishnan, E.V., V.D. Deshmukh, G. Maheshwarudu, J. Joseleen, A.P. Dineshbabu, K.K. Philipose, P.T. Sarada, S.L. Pillai, K.N. Saleela, R. Chakraborthy, G. Dash, C.K. Sajeev, P. Thirumilu, B. Sridhara, Y. Muniyappa, A.D. Sawant, G. Vadiya, R.D. Jhony, J.B. Verma, P.K. Baby, N.P. Unniakrishnan, A. Ramachandran, A. Variamani, A. Palanichany, M. Radhakriashnan \& B. Raju (2012). Prawn fauna (Crustacea: Decapoda) of India - An annotated checklist of the Penaeoid, Sergestoid, Stenopodid and Caridean prawns. Journal of the Marine Biological Association of India 54(1): 50-72; http:// dx.doi.org/10.6024/jmbai.2012.54.1.01697-08

Rajakumaran, P. \& B. Vaseeharan (2014). Survey on Penaeidae shrimp diversity and exploitation in south east coast of India. Fisheries and Aquaculture Journal 5: 103; http://dx.doi.org/10.4172/21503508.1000103

Rao, G.S. (1984). On a collection of two species of pelagic penaeids (Crustacea: Decapoda) from the oceanic waters of the south-west Arabian Sea. Journal of the Marine Biological Association of India 26: 165-166.

Ravindrananth, K. (1989). Taxonomic status of the Coromandel 
shrimp, Parapenaeopsis stylifera coromandelica Alcock (Decapoda, Penaeidae). Crustaceana 57: 258-262.

Shanis, R., K.V. Akhilesh, H. Manjebrayakath, U. Ganga \& N.G.K. Pillai (2012). Shrimps of the family Pandalidae (Caridea) from Indian waters, with new distributional record of Plesionika adensameri (Balss, 1914). Journal of the Marine Biological Association of India 54(1): 45-49; http://dx.doi.org/10.6024/jmbai.2012.54.1.01690-07

Silas, E.G. \& M.S. Muthu (1976). Notes on a collection of penaeid prawns from the Andamans. Journal of the Marine Biological Association of India 18: 78-90.

FAO (2014). Opportunities and Challenges. The Sate of World Fisheries Aquaculture. FAO, 223pp.

Suseelan, C. \& K.H. Mohamed (1968). On the occurrence of Plesionike ensis (A.M.Edw) (Pandalidae, Crustacea) in the Arabian Sea with notes on its biology and fishery potentialities. Journal of Marine Biological Association of India 10(1): 88-94.

Suseelan, C. (1996). Crustacean biodiversity, conservation and management, pp. 41-65. In: Menon, N.D. \& C.S.G. Pillai (eds.). Marine Biodiversity: Conservation and Management. CMFRI, Cochin.

Thomas, M.M. (1979). On a collection of deep sea decapod crustaceans from the Gulf of Mannar. Journal of the Marine Biological Association of India 21: 41-44.

Wood-Mason, J \& A. Alcock (1891). Natural history notes from H.M. Indian Marine Survey Steamer "Investigator", Commander R.F. Hoskyn, R.N., commanding. Series II, No. 1. On the results of deep-sea dredging during the season 1890-1891. The Annals and Magazine of Natural History 6(8): 268-386.

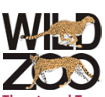

Author Details: VIJAY KUMAR DeEPAK SAMUel is a Scientist at National Centre for Sustainable Coastal Management (NCSCM), Chennai, Tamil Nadu, India. He is a taxonomist specializing on marine molluscs, crustaceans and minor phyla. He is the focal point of the Coastal and Marine Biodiversity Integration Network (CoMBINe) Project. Chemmencheri Ramakrishnan SReeras is a Scientist at NCSCM. His research interests are coral taxonomy and reef ecology. He is a co-investigator of CoMBINe project. PANDIAN KRISHNAN is Scientist in-charge of Coastal \& Marine Resources Conservation Division at NCSCM and is the Principal Investigator of the CoMBINe Project. His research interests are marine ecology, biodiversity conservation and fisheries resource management. CHERMAPANDI Parthiban, Veeramuthu Sekar and Kanagaraj Chamundeeswar are Research Fellows under the CoMBINe project and they specialize in the taxonomy of marine invertebrates. TITUS IMMANUEL is a Project Associate under the project on "Marine biodiversity of Nicobar islands" at ICAR - Central Island Agricultural Research Institute, Port Blair, Andaman. His research interests are taxonomy of marine invertebrates. PATRO SHESDEV, a Scientist at NCSCM is a marine biologist with research interest in biodiversity and ecology of coastal ecosystems. RAMACHANDRAN PuRVaJa is the Division Chair of Coastal \& Marine Resources Conservation Division at NCSCM and is the Coordinator of the CoMBINe Project. Her research interests include coastal zone management, marine ecology and bio-geochemistry. RAMACHANDRAN RAMESH is the Director of National Centre for Sustainable Coastal Management (NCSCM) and is an expert in Integrated Coastal Zone Management.

Author Contribution: VKDS, CRS and PK contributed in checklist verification, interpretation of results and preparing the manuscript. CP, VS and KC contributed in reference collection, preparing the checklist, validating the checklist with WoRMS database and in identifying the issues in the checklist. TI and PS assisted in collating the published literature and updating the checklist. RP and RR critically reviewed and finalized the manuscript.

Tamil abstract:

\section{ஆய்வு சுருக்கம்}

இந்த ஆய்வானது லட்சத்தீவு மற்றும் அந்தமான் நிக்கோபார் தீவுகள் உட்பட இந்திய கடலோர பகுதிகளில் இணைந்து காணப்படுகின்ற கடல் இறால்கள் பற்றிய ஒரு மேம்படுத்தப்பட்ட சரிபார்ப்பு பட்டியலாக தொகுக்கப்பட்டுள்ளது. மொத்தம் 364 இறоால் உயிரினங்கள் 128 இறால் இனங்களின் கீழ் தசக்காலிகளின் (Decapod) வரிசையில் 337 உயிரினங்கள் இருக்கும் இந்த சரிபார்ப்பு பட்டியலில் 27 இனங்கள் சேர்த்த வகைப்படுத்தப்பட்டுள்ளது . பொதுவாக இறால்கள் தசக்காலிகள் வரிசையில் டென்றொப்ராஞ்சியேட்டா (Dendrobranchiata) பிலியோசைமேட்டா (Pleocyemata) என்ற இரு துணை வரிசைகளாக மொத்தம் 364 இனங்கள் 155 (42.6\%) மற்றும் 209(57.4\%) இரு துணை விகிதங்களாக பிர்கப்பட்டுள்ன. இவற்றில் பிலியோசைமேட்டா (Pleocyemata) வரிசையானது, அக்ரிடே (Axiidea), கேரிடே (Caridea) மற்றும் ஸ்டெனோபோடிடே (Stenopodidea) என்ற மூன்று கீழ் வரிசைகளாக பிரிக்கப்பட்டுள்ளது. இவற்றில் கேரிடே (Caridea) 12 குடும்பங்களை கொண்ட பெரிய கீழ் வரிசையாகும். இதில் பினியாயைடே (Penaeoidea) 135 உயிரினங்கள் (38.13\%) பால்மோனியிடே. (Paleaemonidea) 64 உயிரினங்கள் 18.07\% என்ற விகிதத்தில் முக்கிய பங்களிக்கின்றது. மற்ற பெரும்குடும்பங்கள் (superfamilies) $12 \%$ விகிதத்திலும் பிரிசியிலோயிடே (Bresilloidea) மற்றும் சலிடோபோடொய்டே (Psalidopodoidea) ஆகியவை ஒரே ஒரு உயிரினத்தையும் மற்றும் கொண்டுள்ளது. இந்த இறுதி பட்டியிலானது, பட்டியல்கள், தனிக்கட்டுரை, வலைத்தளங்கள், மீன்பிடி அறிக்கைகள் போன்றவைகளிலிருந்து இலக்கிய திறனாய்லு செய்த பிறகு தொகுக்கப்பட்டவையாகும். உயிரினங்களின் அறிவியல் பெயர்கள் அனைத்தும் கடல்வாம் உயிரினங்கள் உலக பதிவேடு (World Register of Marine Species (WoRMS) என்ற தகவல் மூலம் சரிபார்க்கப்பட்டவையாகும். இந்த ஆய்வில் மொத்கம் 25 1 உயிரினின்களின் பிழைகள் மேம்படுத்தப்பட்டு 6 உயிரினங்கள் நீக்கப்பட்டுள்ளன. 



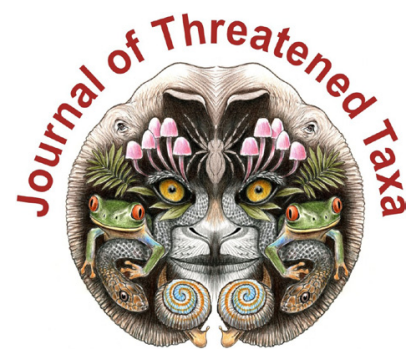

All articles published in the Journal of Threatened Taxa are registered under Creative Commons Attribution 4.0 International License unless otherwise mentioned. JoTT allows unrestricted use of articles in any medium, reproduction and distribution by providing adequate credit to the authors and the source of publication.

\author{
ISSN $0974-7907$ (Online); ISSN 0974-7893 (Print) \\ July 2016 | Vol. 8 | No. 7 | Pages: 8953-9052 \\ Date of Publication: 26 July 2016 (Online \& Print) \\ DOI: 10.11609/jott.2016.8.7.8953-9052 \\ www.threatenedtaxa.org
}

Article

Bats (Mammalia: Chiroptera) of the southeastern Truong Son Mountains, Quang Ngai Province, Vietnam

-- Nguyen Truong Son, Thomas J. O'Shea, Jeffery A. Gore, Csorba Gabor, Vuong Tan Tu, Tatsuo Oshida, Hideki Endo \& Masaharu Motokawa, pp. 8953-8969

\section{Communication}

An assessment of human-elephant conflict and associated ecological and demographic factors in Nilambur, Western Ghats of Kerala, southern India

-- C.K. Rohini, T. Aravindan, P.A. Vinayan, M. Ashokkumar \& K.S. Anoop Das, Pp. 8970-8976

Review

An updated checklist of shrimps on the Indian coast -- Vijay Kumar Deepak Samuel, Chemmencheri Ramakrishnan Sreeraj, Pandian Krishnan, Chermapandi Parthiban, Veeramuthu Sekar, Kanagaraj Chamundeeswari, Titus Immanuel, Patro Shesdev, Ramachandran Purvaja \& Ramachandran Ramesh, Pp. 8977-8988

\section{View Point}

Can philately sensitise people to wildlife / conservation? An introduction to thematic philately and a visual treatise concerning the variety of philatelic material available on owls (Aves: Strigiformes)

-- M. Eric Ramanujam, Pp. 8989-9003

\section{Short Communications}

Noteworthy additions to the flora of Uttarakhand, western Himalaya, India

-- Ishwari D. Rai, Gajendra Singh \& Gopal S. Rawat, Pp. 9004-9008

Seed germination studies on Gymnacranthera canarica (King) Warb. - a Vulnerable tree species of a highly threatened Myristica swamp ecosystem

-- K. Keshavachandra \& G. Krishnakumar, Pp. 9009-9013

A first note on foliicolous lichens of Assam, India

-- Pooja Gupta \& G.P. Sinha, Pp. 9014-9023
Notes

A recent record of the Indo-Pacific Humpback Dolphin Sousa chinensis (Osbeck, 1765), (Mammalia: Cetartiodactyla: Delphinidae) from the western shores of Kachchh, Gujarat, India

-- Devanshi Kukadia, Mayurdan K. Gadhavi, N. Gokulakannan, G.V. Gopi, Gautam Talukdar \& K. Sivakumar, Pp. 9024-9026

A confirmation of the occurrence of Euploea sylvester hopei Felder \& Felder, 1865 (Double-branded Blue Crow) from Kaptai National Park, Rangamati District, Bangladesh

-- Tahsinur Rahman Shihan, Pp. 9027-9029

A century later: Tricolored Pied Flat Coladenia indrani uposathra Fruhstorfer, 1911 (Hesperiidae: Pyrginae) and Crenulate Oakblue Apporasa atkinsoni Hewitson, 1869 (Lycaenidae: Theclinae) reported from Manipur, India -- Baleshwor Singh Soibam, Harmenn Huidrom \& Jatishwor Singh Irungbam, Pp. 9030-9033

On the distribution of Aeshna petalura Martin, 1908 (Odonata: Anisoptera: Aeshnidae) in the Indian subcontinent

-- R. Babu \& G. Srinivasan, Pp. 9034-9037

Notes on the occurrence of Mortonagrion aborense Laidlaw, 1914 (Odonata: Coenagrionidae) from lower West Bengal, India -- Arajush Payra \& Ashish D. Tiple, Pp. 9038-9041

First record of Speculitermes chadaensis Chatterjee \& Thapa, 1964 (Isoptera: Termitidae) from the Western Ghats, India -- Poovoli Amina, K. Rajmohana \& K.V. Bhavana, Pp. 9042-9044

A first report of egg parasitism in the Tropical Tasar Silkworm Antheraea mylitta (Drury) occurring on cashew -- K. Vanitha \& S. Santhosh, Pp. 9045-9047

Gentiana saginoides Burkill (Magnoliopsida: Gentianales: Gentianaceae) rediscovered from Sunderdhunga Valley in Uttarakhand 155 years after description: notes on its population status

-- Dharmendra S. Rawat, Charan S. Rana, Harish Singh \& Manish Karnatak, Pp. 9048-9052
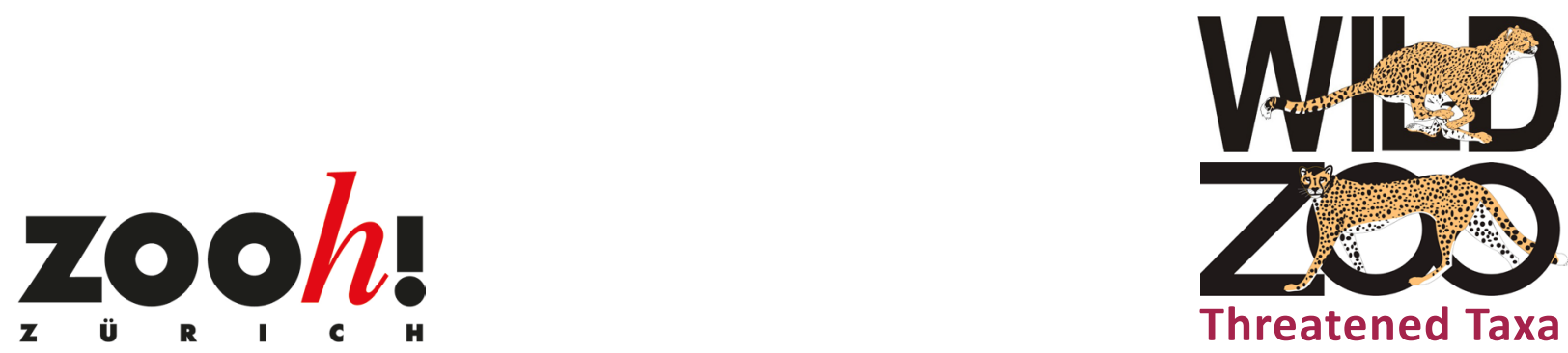